\title{
Deformation of a hydrophobic ferrofluid droplet suspended in a viscous medium under uniform magnetic fields
}

\author{
S. AFKHAMI $I^{1,2}$, A. J. TYLER ${ }^{3}, Y$. RENARDY $Y^{2}$, \\ M. RENARDY ${ }^{2}$, T. G. St. PIERRE ${ }^{3}$, R. C. WOODWAR D \\ AND J. S. RIFF LE E $^{4}$ \\ ${ }^{1}$ Department of Mathematical Sciences, New Jersey Institute of Technology, Newark, NJ 07102, USA \\ ${ }^{2}$ Department of Mathematics, Virginia Tech, Blacksburg, VA 24061, USA \\ ${ }^{3}$ School of Physics, M013, The University of Western Australia, Crawley, WA 6009, Australia \\ ${ }^{4}$ Department of Chemistry and the Macromolecules and Interfaces Institute, Virginia Tech, Blacksburg, \\ VA 24061, USA
}

(Received 29 April 2010; revised 29 June 2010; accepted 30 June 2010; first published online 8 September 2010)

The effect of applied magnetic fields on the deformation of a biocompatible hydrophobic ferrofluid drop suspended in a viscous medium is investigated numerically and compared with experimental data. A numerical formulation for the time-dependent simulation of magnetohydrodynamics of two immiscible nonconducting fluids is used with a volume-of-fluid scheme for fully deformable interfaces. Analytical formulae for ellipsoidal drops and near-spheroidal drops are reviewed and developed for code validation. At low magnetic fields, both the experimental and numerical results follow the asymptotic small deformation theory. The value of interfacial tension is deduced from an optimal fit of a numerically simulated shape with the experimentally obtained drop shape, and appears to be a constant for low applied magnetic fields. At high magnetic fields, on the other hand, experimental measurements deviate from numerical results if a constant interfacial tension is implemented. The difference can be represented as a dependence of apparent interfacial tension on the magnetic field. This idea is investigated computationally by varying the interfacial tension as a function of the applied magnetic field and by comparing the drop shapes with experimental data until a perfect match is found. This estimation method provides a consistent correlation for the variation in interfacial tension at high magnetic fields. A conclusion section provides a discussion of physical effects which may influence the microstructure and contribute to the reported observations.

Key words: drops, ferrofluid, magnetohydrodynamics, volume-of-fluid method

\section{Introduction}

Many recent developments in the synthesis and characterization of ferrofluids are motivated by biomedical applications, such as the delivery of therapeutic drugs to specific parts of the body (Voltairas, Fotiadis \& Massalas 2001; Voltairas, Fotiadis \& 
(a)

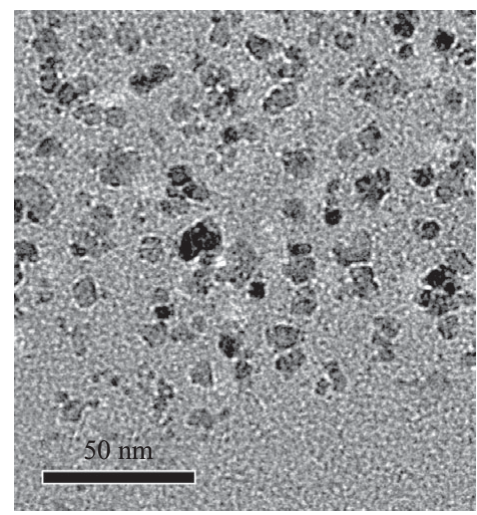

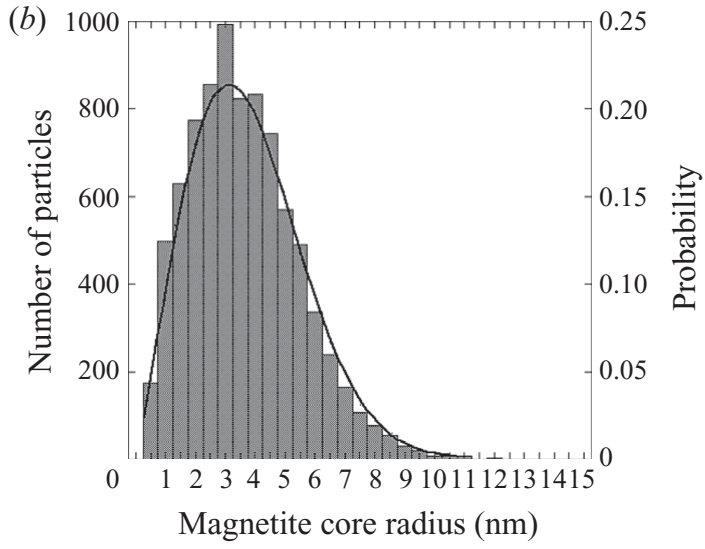

Figure 1. Reproduced from figures 4.2 and 4.3 of Mefford (2007), and figures 2 and 3 of Mefford et al. (2008b). (a) Representative TEM image of PDMS-magnetite particles. (b) Histogram of the radii of the magnetite cores.

Michalis 2002; Holligan, Gillies \& Dailey 2003; Dames et al. 2007; Liu et al. 2007). In particular, we focus on the behaviour of a hydrophobic ferrofluid drop which is being synthesized for the potential treatment of retinal detachment (Mefford et al. 2007, $2008 a, b)$. The ferrofluid consists of magnetite nanoparticles coated with biocompatible polydimethylsiloxane (PDMS) oligomers, with the advantages of a narrow size distribution, and colloidal stability. The PDMS-nanoparticle complex is manufactured without the addition of a carrier fluid, which helps to minimize phase separation in applied fields. Figure 1 shows $(a)$ a sample transmission electron microscopy (TEM) image of the magnetite nanoparticles at 300000 times magnification and $(b)$ the particle size distribution, with an arithmetic mean for core diameters at $7.2 \mathrm{~nm}$ and standard deviation of $3.6 \mathrm{~nm}$. A starting point for the prediction and control of a ferrofluid droplet in complex biomedical applications is the simplified model of a ferrofluid droplet suspended in a viscous medium, in an applied magnetic field. A magnetic drop freely suspended in a surrounding viscous medium elongates in the direction of the uniform magnetic field and assumes a stable equilibrium configuration. The axial ratio increases with an increase of the magnetic field strength. Examples of an experimentally observed PDMS ferrofluid drop suspended in glycerol in uniform magnetic fields are depicted in figure 2. The governing equations for this system are stated in $\S 2$.

If a spherical shape is assumed for the ferrofluid drop, then the imposed magnetic field leads to a mismatch in the normal stress condition at the interface (Arkhipenko, Barkov \& Bashtovoi 1978; Drozdova, Skrobotova \& Chekanov 1979). The excess pressure forces the drop to elongate in the direction of the field. A problem equivalent to that of the ferrofluid drop arises in the deformation of a dielectric drop in an applied electric field. An extensive body of theoretical work has evolved, under the assumption that the deformed drop has a spheroidal shape. In this case, a closed-form solution for the electric field is found for the case of a uniform applied field. This leaves the normal stress balance at the interface yet to be satisfied, and one free parameter for the drop shape, namely the aspect ratio. We cannot satisfy the normal stress balance at every point, but we can satisfy it selectively to complete an approximate solution. One option is to satisfy the normal stress balance at the poles and equator of the drop, and another is to satisfy it in some averaged sense. Taylor (1964) investigated 
(a)

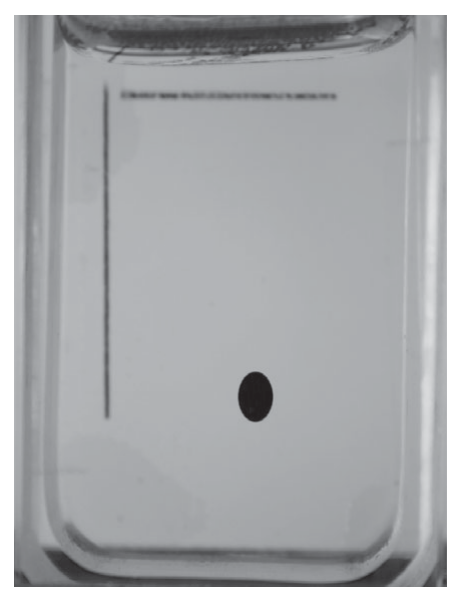

(b)

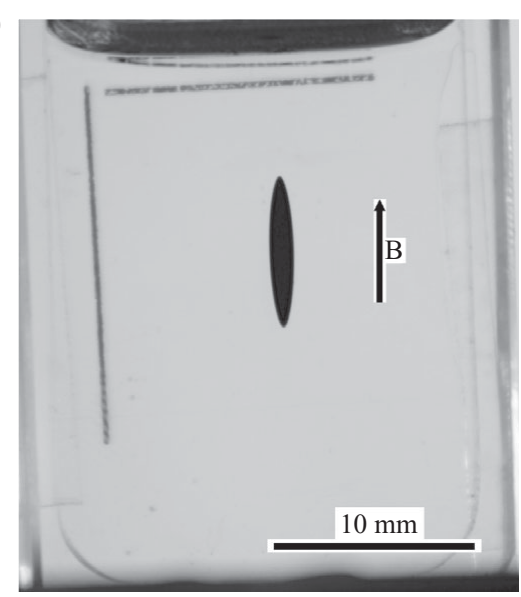

FIGURE 2. Experimental photographs of the deformation of a PDMS ferrofluid drop in a viscous medium under applied uniform magnetic fields. (a) $H_{o}=6.38 \mathrm{kA} \mathrm{m}^{-1}$ and (b) $638.21 \mathrm{kA} \mathrm{m}^{-1}$. Images were collected using the equipment shown in figure 11 (Tyler 2008).

a perfectly conducting spheroid in a uniform electric field by satisfying the normal stress balance at the pole and the equator. This two-point approximation revealed that the drop deformation as a function of the electric field may be multi-valued above a certain electric field threshold. Garton \& Krasucki (1964) showed that a drop suspended in an insulating liquid under an applied electric field assumes the shape of a prolate spheroid in the direction of the field whenever the permittivity is different from that of the surrounding medium. Miksis (1981) studied a system of nonlinear integro-differential equations from a boundary-integral formulation for an axisymmetric dielectric drop in a uniform electric field. He showed that a nearly prolate spheroidal shape is achieved for dielectric constants below a critical value. A slender-body approximation of Sherwood (1991) predicted more pointed ends than those of a spheroid for the case of a strong imposed field. Wohlhuter \& Basaran (1992) used a Galerkin finite element method to address the stability of a linearly polarizable dielectric drop, pendant or sessile on a plate, subjected to an applied electric field. Their free boundary problem comprised the magnetic Young-Laplace equation for the drop shape coupled with the Maxwell equations. Feng \& Scott (1996) performed a computational study of the influence of the electric field strength on drop shape.

For the case of a ferrofluid drop suspended in a non-magnetizable medium in an externally applied uniform magnetic field, the assumption of a spheroidal shape led to a corresponding closed-form solution for the magnetic field. The aspect ratio of the drop was a free parameter, which was determined from the balance of linear moments of force (Cebers 1985; Blums, Cebers \& Maiorov 1997), or, equivalently, the minimization of the sum of the magnetostatic energy and the surface tension energy (Bacri \& Salin 1982). The equilibrium drop aspect ratio was found to continuously increase with magnetic field strength at low values of the permeability ratio of the drop to the surrounding liquid. Above a critical permeability ratio, the drop aspect ratio displayed an S-shaped curve dependence on the applied field, so that three equilibrium solutions occurred above a threshold magnetic field, two of which were stable and one unstable. Here, the aspect ratio increased continuously up to a threshold field strength, and then jumped to the higher solution branch. These 
stability results were retrieved in the analysis of Wohlhuter \& Basaran (1992) for linearly polarizable drops. The numerical study of a nonlinearly polarizable ferrofluid drop showed similar behaviour to linearly polarizable drops at low magnetic field strengths when drop deformations were not substantial (Basaran \& Wohlhuter 1992). Lavrova et al. $(2004,2006)$ also considered nonlinearly magnetizable ferrofluid drops and used a finite element method in which the governing equations of the magnetic liquid were coupled by the force balance at the interface and the surface tension was applied as a boundary condition at the interface. Their numerical results of drop equilibrium shapes were in accordance with the theory of Bacri \& Salin (1982). Korlie et al. (2008) used a volume-of-fluid (VOF) method to directly simulate a bubble of a non-magnetic fluid rising through a linearly magnetic ferrofluid. In their numerical algorithm, the magnetic force was defined as a jump in magnetic normal stress across the interface and was applied as a magnetic interfacial force acting only as a normal force on the interface. Afkhami et al. $(2008 a, b)$ addressed the time-dependent simulation of a nonlinearly magnetizable ferrofluid drop moving in a viscous medium under an applied non-uniform magnetic field. The full Navier-Stokes equations and coupled magnetostatic equations were solved over the entire flow field.

An equilibrium state under a uniform magnetic field is achieved via the competition between capillary and magnetic stresses. The calculation of magnetic stresses at the interface requires the knowledge of the drop shape. The coupling of the interfacial shape and the variation of the magnetic field hinder the development of analytical exact solutions except in limiting cases, such as the prolate ellipsoid for low magnetic fields (Tyatyushkin \& Verlarde 2001). In $\S 3$, we focus on an axisymmetric droplet that is small compared with the distance to the boundaries, and retrieve the available exact solutions and perturbation solutions for equilibria.

At sufficiently high fields, shape transitions such as conical tips may occur (Bacri \& Salin 1982; Stone, Lister \& Brenner 1999; Lavrova et al. 2006). Our numerical algorithm to handle such regimes includes modifications to the discretization scheme for the magnetic stress tensor and the averaging scheme for the magnetic fluid properties, within the framework already detailed elsewhere (Afkhami et al. 2008a). Section 4 is a brief summary of the numerical methodology. In $\S 5$, we report direct numerical simulations of the deformation of a hydrophobic ferrofluid drop suspended in a viscous medium under uniform magnetic fields, for the experimental system exemplified by figure 2 . An interesting outcome is the dependence of interfacial tension on magnetic field strength. Concluding remarks are given in $\S 6$.

\section{Governing equations}

The coupled motion of a ferrofluid drop suspended in a viscous fluid is governed by the Maxwell equations, the Navier-Stokes equations and a constitutive relationship for the magnetic induction $\boldsymbol{B}$, magnetic field $\boldsymbol{H}$ and magnetization $\boldsymbol{M}$. The magnetostatic Maxwell equations for a non-conducting ferrofluid are, in SI units,

$$
\begin{gathered}
\nabla \cdot \boldsymbol{B}=0, \quad \nabla \times \boldsymbol{H}=0, \\
\boldsymbol{B}(\boldsymbol{x}, t)= \begin{cases}\mu_{d} \boldsymbol{H} & \text { in the ferrofluid drop, } \\
\mu_{m} \boldsymbol{H} & \text { in the matrix, }\end{cases}
\end{gathered}
$$

where $\mu_{d}$ denotes the permeability of the drop, and $\mu_{m}$ is the permeability of the matrix fluid. For our application, the matrix fluid is glycerol, which has a permeability very close to that for a vacuum, $\mu_{o}$. Therefore, we shall set $\mu_{m}=\mu_{o}$ throughout the 
mathematical analysis. A magnetic scalar potential $\phi$ is defined by $\boldsymbol{H}=\nabla \phi$, and satisfies

$$
\nabla \cdot(\mu \nabla \phi)=0
$$

We will assume initially that the magnetization is a linear function of the magnetic field given by $\boldsymbol{M}=\chi \boldsymbol{H}$, where $\chi=\left(\mu / \mu_{o}-1\right)$ is the magnetic susceptibility. The magnetic induction $\boldsymbol{B}$ is therefore $\boldsymbol{B}=\mu_{o}(\boldsymbol{H}+\boldsymbol{M})=\mu_{o}(1+\chi) \boldsymbol{H}$. The fluid equation of motion is

$$
\rho \frac{\mathrm{d} \boldsymbol{u}}{\mathrm{d} t}=-\nabla p+\nabla \cdot 2 \eta \boldsymbol{D}+\boldsymbol{F}_{s}+\nabla \cdot \boldsymbol{\tau}_{m},
$$

where $\boldsymbol{F}_{s}$ denotes a body force, $p$ is pressure, $\boldsymbol{u}$ is velocity, $\boldsymbol{D}=\left(\boldsymbol{\nabla} \boldsymbol{u}+(\nabla \boldsymbol{u})^{\mathrm{T}}\right) / 2$ is the rate of deformation tensor where T denotes the transpose, $\eta$ is viscosity, $\rho$ is density and $\boldsymbol{\tau}_{m}$ is the magnetic stress tensor. Let $\boldsymbol{\tau}=-p \boldsymbol{I}+2 \eta \boldsymbol{D}+\boldsymbol{\tau}_{m}$, where $\boldsymbol{I}$ denotes the identity operator.

The magnetic stress tensor of an incompressible, isothermal, linearly magnetizable medium is

$$
\boldsymbol{\tau}_{m}=-\frac{\mu}{2} H^{2} \boldsymbol{I}+\mu \boldsymbol{H} \boldsymbol{H}^{\mathrm{T}},
$$

where $H=|\boldsymbol{H}|$. Let $\llbracket x \rrbracket$ denote the difference $x_{\text {matrix }}-x_{\text {drop }}$ at the interface between the ferrofluid drop and the external liquid. Let $\boldsymbol{n}$ denote the unit normal outwards from the interface, and $\boldsymbol{t}_{1}, \boldsymbol{t}_{2}$ denote the orthonormal tangent vectors. We require $(a)$ the continuity of velocity, the normal component of magnetic induction, the tangential component of the magnetic field, the tangential component of surface stress,

$$
\llbracket \boldsymbol{u} \rrbracket=0, \quad \boldsymbol{n} \cdot \llbracket \boldsymbol{B} \rrbracket=0, \quad \boldsymbol{n} \times \llbracket \boldsymbol{H} \rrbracket=\mathbf{0}, \quad\left[\left[\boldsymbol{t}_{1}^{\mathrm{T}} \boldsymbol{\tau} \boldsymbol{n}\right]\right]=0, \quad\left[\left[\boldsymbol{t}_{2}^{\mathrm{T}} \boldsymbol{\tau} \boldsymbol{n}\right]\right]=0,
$$

and $(b)$ the jump in the normal component of stress is balanced by capillary effects,

$$
\llbracket \boldsymbol{n}^{\mathrm{T}} \boldsymbol{\tau} \boldsymbol{n} \rrbracket=\sigma \kappa,
$$

where $\sigma$ is the interfacial tension and $\kappa=-\nabla \cdot \boldsymbol{n}$ is the sum of principal curvatures. We refer the reader to Rosensweig (1985) and Afkhami et al. (2008a) for details of the magnetic contribution to the interface conditions.

The density and the viscosity of the ferrofluid drop are matched with the surrounding liquid. The important dimensionless parameter is the magnetic Bond number,

$$
B o_{m}=\frac{\mu_{o} H_{o}^{2}}{\sigma \kappa_{o}}
$$

where $H_{o}$ is the applied magnetic field intensity and $\kappa_{o}$ is the curvature of the undeformed drop of radius $R_{o}$. The magnetic Bond number is the ratio of magnetic force to interfacial tension force. A Reynolds number for the transient flow to equilibrium is $R e=\left(\rho \mu_{o} H_{o}^{2} R_{o}^{2}\right) / \eta^{2}$, which represents inertia multiplied by magnetic force divided by the square of the viscous drag. This parameter is small throughout this paper.

\section{Drop equilibrium shapes}

Analytical formulae for limiting cases are useful for validating the experimental data, as well as numerical results for regimes where experimental data are not available. In this section, we develop and review the analytical solution for a ferrofluid drop in equilibrium, suspended in a non-magnetizable viscous matrix. Both the drop 
and the surrounding liquid are non-conducting, with no free charges at the surface. In such a case, the magnetic force is a surface force acting on the interface of the two liquids and is solely induced by the difference in permeability of the two liquids. We further assume a linear magnetic material for the analytical formulae developed in this section, i.e. constant $\mu_{d}$ and $\chi$.

In the absence of fluid motion, the shape of the interface is governed by a balance between interfacial tension stress and magnetic stress. Magnetic interfacial force can then be defined in terms of the jump in magnetic normal stress at the interface. The force balance in the direction normal to the interface in the presence of an applied magnetic field is given by the magnetic Young-Laplace equation

$$
-\llbracket p \rrbracket+\left[\left[\frac{\mu}{2}\left((\boldsymbol{H} \cdot \boldsymbol{n})^{2}-(\boldsymbol{H} \cdot \boldsymbol{t})^{2}\right)\right]\right]=\sigma \kappa .
$$

\subsection{Spherical ferrofluid drop in a uniform magnetic field}

In spherical coordinates with axisymmetry, Laplace's equation for the magnetic potential $\phi$ is $(\partial / \partial r)\left(r^{2}(\partial \phi / \partial r)\right)+(1 / \sin \theta)(\partial / \partial \theta)(\sin \theta(\partial \phi / \partial \theta))=0$, where $r$ is the radial distance from the drop centre and $\theta$ is the polar angle. Separation of variables yields Legendre polynomials for the $r$-dependence, only the first of which is relevant:

$$
\phi(r, \theta)= \begin{cases}\mathscr{A} r \cos \theta & r \leqq R_{o} \\ \left(\mathscr{C} r+\mathscr{D} / r^{2}\right) \cos \theta & r>R_{o}\end{cases}
$$

where $R_{o}$ is the radius of the spherical drop. The associated magnetic field $\boldsymbol{H}=(\partial \phi / \partial r) \boldsymbol{e}_{r}+(1 / r)(\partial \phi / \partial \theta) \boldsymbol{e}_{\theta}$ is

$$
\boldsymbol{H}(r, \theta)= \begin{cases}\mathscr{A}\left(\cos \theta \boldsymbol{e}_{r}-\sin \theta \boldsymbol{e}_{\theta}\right), & r \leqq R_{o}, \\ \left(\mathscr{C}-2 \mathscr{D} / r^{3}\right) \cos \theta \boldsymbol{e}_{r}-\left(\mathscr{C}+\mathscr{D} / r^{3}\right) \sin \theta \boldsymbol{e}_{\theta}, & r>R_{o} .\end{cases}
$$

The far-field condition is a uniform applied field $H_{o}\left(\cos \theta \boldsymbol{e}_{r}-\sin \theta \boldsymbol{e}_{\theta}\right)$. Thus, $\mathscr{C}=H_{o}$. Because the tangential component of $\boldsymbol{H}$ and the normal component of $\boldsymbol{B}$ are continuous across the interface, $\mathscr{A}$ and $\mathscr{D}$ are

$$
\mathscr{A}=\frac{3 \mu_{o}}{\mu_{d}+2 \mu_{o}} H_{o}, \quad \mathscr{D}=-\frac{\mu_{d}-\mu_{o}}{\mu_{d}+2 \mu_{o}} R_{o}^{3} H_{o} .
$$

The pressure jump across the interface of a spherical ferrofluid drop of radius $R_{o}$ in a uniform magnetic field is in general non-uniform and is a function of the polar angle $\theta$. Equation (3.1) is

$$
-\llbracket p \rrbracket=-\frac{\mu_{o}}{2}\left(H_{m n}^{2}-H_{m t}^{2}\right)+\frac{\mu_{d}}{2}\left(H_{\mathrm{d} n}^{2}-H_{\mathrm{d} t}^{2}\right)+2 \sigma / R_{o},
$$

where

$$
\begin{aligned}
& H_{\mathrm{d} n}=\left(\frac{\mu_{o}}{\mu_{d}}\right) H_{m n}=\frac{3 \mu_{o}}{\mu_{d}+2 \mu_{o}} H_{o} \cos \theta \\
& H_{\mathrm{d} t}=H_{m t}=-\frac{3 \mu_{o}}{\mu_{d}+2 \mu_{o}} H_{o} \sin \theta
\end{aligned}
$$

the subscript $m$ refers to the matrix liquid, and the second subscripts $n$ and $t$ refer to the normal and tangential components, respectively.

\subsection{Ferrofluid drop deformation in an applied uniform magnetic field}

The deformation of a ferrofluid drop in an applied uniform magnetic field is considered theoretically with axisymmetry. When the drop is ellipsoidal, we can take 
advantage of the exact solution for the case of a uniform magnetic field inside the drop in order to develop an analytical approximation (Garton \& Krasucki 1964; Taylor 1964; Rallison 1980; Dodgson \& Sozou 1987; Sherwood 1991). When the shape of the drop is imposed, the set of governing equations is overdetermined, so that the normal stress balance cannot be satisfied over the entire surface of the drop. Hence, a decision must be made for one further approximation in order to solve for the aspect ratio. Taylor (1964) chose to satisfy the normal stress balance at the poles and equator. Another possibility is to balance linear moments of force (Cebers 1985; Blums et al. 1997) or, equivalently, minimize the sum of magnetic and surface energy, subject to the constraint that the drop shape remains ellipsoidal (Bacri \& Salin 1982). These approximations agree for drops at low to moderate deformations, and we shall see that they agree with the full numerical simulations. For larger deformations, the approximate theories begin to deviate from each other, as expected. The experiments we shall discuss below are well outside the range where the approximate theories start to deviate from each other, and for purposes of validating our code, we shall proceed with the simpler method of imposing the normal stress balance along the two major principal axes.

Landau \& Lifshitz (1984) gave an analytical expression for the uniform field inside an ellipsoid. The relationship between $\boldsymbol{H}=(0,0, H), \boldsymbol{B}=(0,0, B)$, and the applied uniform far-field magnetic field $\boldsymbol{H}_{o}=\left(0,0, H_{o}\right)$ is $[\zeta H+\xi B]_{i n}=H_{o}$, where the coefficients $\zeta$ and $\xi$ depend only on the shape of the ellipsoid and the subscript in refers to inside the ellipsoid. The existence of such a relation is based on the fact that the field inside an axisymmetric drop is uniform and follows from the form of the boundary conditions discussed in $\S 3.1$. The magnetic field inside a prolate spheroid is found to be

$$
(1-k) \mu_{\text {out }} H_{\text {in }}+k B_{\text {in }}=\mu_{\text {out }} H_{o},
$$

where the subscript out denotes the fluid outside the drop. Here $k$ is the demagnetizing factor:

$$
k=\left(\frac{1-E^{2}}{2 E^{3}}\right)\left(\ln \frac{1+E}{1-E}-2 E\right),
$$

where $E=\sqrt{1-a^{2} / b^{2}}$ is the eccentricity, $2 a$ is the minor axis and $2 b$ is the major axis (the direction of the applied magnetic field, denoted by the $z$-direction). Since $B=\mu H$, the field interior of a spheroid drop becomes

$$
H_{\text {in }}=\frac{\mu_{\text {out }} H_{o}}{(1-k) \mu_{\text {out }}-k \mu_{\text {in }}} \text {. }
$$

Note that for a spherical drop, $k=1 / 3$, which recovers the magnetic field calculated in $\S 3.1$. Now consider a drop of permeability $\mu_{d}$ immersed in a matrix liquid of permeability $\mu_{m}$ and subjected to a uniform field between parallel plates of intensity $H_{o}$. Let us now write the force balance under equilibrium conditions along the two principal axes: at the pole,

$$
p_{m}-p_{d}-\llbracket \boldsymbol{n} \cdot \boldsymbol{\tau}_{m} \cdot \boldsymbol{n} \rrbracket_{\text {pole }}+\sigma \kappa_{\text {pole }}=0,
$$

and at the equator,

$$
p_{m}-p_{d}-\llbracket \boldsymbol{n} \cdot \boldsymbol{\tau}_{m} \cdot \boldsymbol{n} \rrbracket_{\text {equator }}+\sigma \kappa_{\text {equator }}=0,
$$

where $p_{m}$ and $p_{d}$ are uniform pressures in the drop and matrix, respectively, $\llbracket \boldsymbol{n} \cdot \boldsymbol{\tau} \cdot \boldsymbol{n} \rrbracket$ is the normal magnetic stress jump across the interface, and $\kappa_{\text {pole }}$ and $\kappa_{\text {equator }}$ are the interfacial curvatures at the pole and the equator of the drop. 


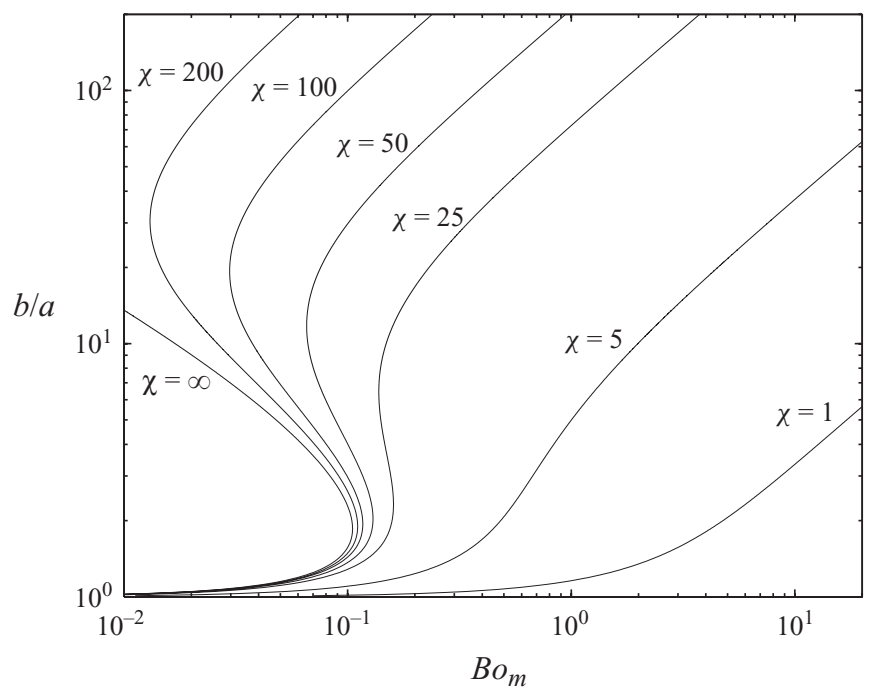

FIGURE 3. Drop aspect ratio $b / a$ at equilibrium, where $b$ is the semi-major axis and $a$ is the semi-minor axis, shown as a function of the magnetic Bond number $B o_{m}$ for $\chi$ varying from 1 to $\infty$; cf. (3.16).

For a prolate spheroid with major axis $a$ and minor axis $b$, the result of this analysis yields the following relationship between the aspect ratio and the field inside the drop:

$$
\frac{\left(\mu_{m}-\mu_{d}\right)^{2}}{\mu_{m}} \frac{R_{o} H_{d}^{2}}{2 \sigma}=\left(\frac{b}{a}\right)^{1 / 3}\left(2 \frac{b}{a}-\left(\frac{b}{a}\right)^{-2}-1\right) .
$$

The drop retains axisymmetry about the direction of the field in the shape of a prolate spheroid, and therefore (3.8) shows that the magnetic field inside the drop, $\boldsymbol{H}_{d}$, must be uniform and differs only in magnitude from the applied field, $\boldsymbol{H}_{o}$. Thus,

$$
H_{d}=\frac{\mu_{m}}{(1-k) \mu_{m}+k \mu_{d}} H_{o} \text {. }
$$

Substitution in (3.13) gives

$$
\frac{\left(\mu_{m}-\mu_{d}\right)^{2}}{\mu_{m}}\left(\frac{\mu_{m}}{(1-k) \mu_{m}+k \mu_{d}} H_{o}\right)^{2} \frac{R_{o}}{2 \sigma}=\left(\frac{b}{a}\right)^{1 / 3}\left(2 \frac{b}{a}-\left(\frac{b}{a}\right)^{-2}-1\right) .
$$

This simplifies with the use of (2.8), where the curvature for the undeformed sphere is $\kappa_{o}=2 / R_{o}$, and $\chi=\left(\mu_{d}-\mu_{m}\right) / \mu_{m}$ to

$$
B o_{m}=\left[\frac{1}{\chi}+k\right]^{2}\left(\frac{b}{a}\right)^{1 / 3}\left(2 \frac{b}{a}-\left(\frac{b}{a}\right)^{-2}-1\right) .
$$

Figure 3 displays the aspect ratio at equilibrium as a function of the magnetic Bond number at fixed values of the magnetic susceptibility $\chi$. The figure shows that at a low permeability ratio, such as the case $\chi=1$, the drop deforms continuously and gradually as the magnetic field is increased. We shall address experimental data for this behaviour in $\S 5$. This figure also shows that there is a critical permeability ratio $\mu_{d} / \mu_{m}$, above which the aspect ratio versus magnetic field strength is an S-shaped 


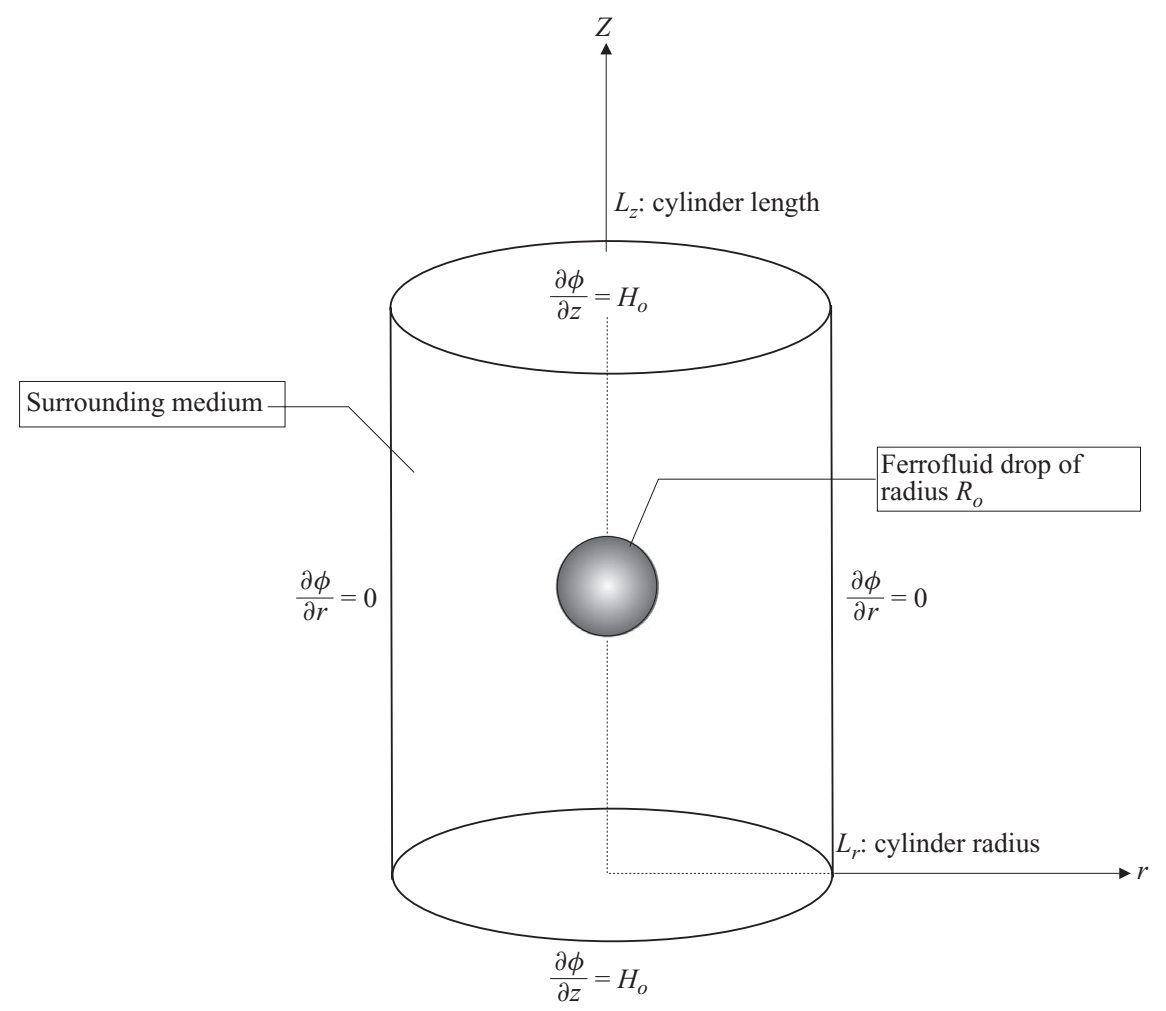

FIGURE 4. Schematic of the initial configuration. The computational domain is $0 \leqslant z \leqslant L_{z}$, $0 \leqslant r \leqslant L_{r}$. Initially, a spherical superparamagnetic ferrofluid drop of radius $R$ is centred in the domain. The boundary conditions on the magnetic field are shown at the boundaries.

curve. The aspect ratio is then multi-valued above a critical magnetic field strength. An example is the case $\chi=50$, for which there is only one equilibrium shape for a low magnetic field strength, three aspect ratios for an intermediate magnetic field strength, two of which are stable and one of which is unstable, and for high magnetic field strengths, there is only a long slender shape. These features are reminiscent of the experimental results of Bacri \& Salin (1982), for which the permeability ratio is roughly 40 and the field varies from $0.056 \mathrm{kA} \mathrm{m}^{-1}$ to $0.112 \mathrm{kA} \mathrm{m}^{-1}$. In their case, the surface tension is $6.5 \times 10^{-4} \mathrm{mN} \mathrm{m}^{-1}$, the radius is of the order of microns, and the prolate spheroidal drop transitions at a critical magnetic field strength to an aspect ratio of roughly 20 with what appear to be conical ends. We shall show such a transition with direct numerical simulations in $\S 4.2$.

\section{Numerical methodology}

The initial configuration for the computational study of a ferrofluid drop suspended in a non-magnetizable viscous medium is shown in figure 4. We refer to the numerical algorithm detailed by Afkhami et al. $(2008 a)$, but with the following modifications.

(a) The stress tensor is modified to expedite the direct validation with the formulae in $\S 3$ for the equilibrium pressure jump across the interface. The spatial discretization is given in the Appendix. 
(b) A harmonic average of the magnetic permeability is more accurate than a simple average for the simulation of highly elongated drops, as in the case of the heat equation (Patankar 1980).

Continuum equations. In summary, the VOF method represents each liquid with a colour function:

$$
C(r, z, t)= \begin{cases}0 & \text { in the surrounding medium, } \\ 1 & \text { in the ferrofluid drop, }\end{cases}
$$

which is advected by the flow. The position of the interface is reconstructed from $C(r, z, t)$. In the equation of motion (2.4), $\boldsymbol{F}_{s}$ denotes the continuum body force due to interfacial tension:

$$
\boldsymbol{F}_{s}=\sigma \kappa \delta_{S} \boldsymbol{n},
$$

where $\boldsymbol{n}=\nabla C /|\nabla C|$ and $\delta_{S}=|\nabla C|$ is the delta-function at the interface. The magnetic stress tensor $\boldsymbol{\tau}_{m}$ is $\mu\left(\boldsymbol{H} \boldsymbol{H}^{\mathrm{T}}-\left(H^{2} \boldsymbol{I} / 2\right)\right)$ (the Appendix). The equation of motion becomes

$$
\rho \frac{\mathrm{d} \boldsymbol{u}}{\mathrm{d} t}=-\nabla p+2 \nabla \cdot \eta \boldsymbol{D}+\boldsymbol{F}_{s}+\nabla \cdot\left[\mu\left(\boldsymbol{H} \boldsymbol{H}^{\mathrm{T}}-\frac{1}{2} H^{2} \boldsymbol{I}\right)\right] .
$$

The magnetic potential $\phi$ in axisymmetric cylindrical coordinates satisfies (2.3):

$$
\frac{1}{r} \frac{\partial}{\partial r}\left(\mu r \frac{\partial \phi}{\partial r}\right)+\frac{\partial}{\partial z}\left(\mu \frac{\partial \phi}{\partial z}\right)=0 .
$$

Discretization. The computational domain, $\left[0, L_{r}\right] \times\left[0, L_{z}\right]$, is uniformly discretized with cell size $\Delta \times \Delta$. The discretized colour function in a given cell now represents the volume fraction of the ferrofluid component. The advection of the volume-fraction function is Lagrangian, and the piecewise linear interface reconstruction scheme (PLIC) is used to calculate the interface position at each time step. A volume average interpolation is used to calculate the density and viscosity and a weighted harmonic mean interpolation is used to compute the magnetic permeability at the computational cell:

$$
\rho=C \rho_{d}+(1-C) \rho_{m}, \quad \eta=C \eta_{d}+(1-C) \eta_{m}, \quad \frac{1}{\mu}=\frac{C}{\mu_{d}}+\frac{1-C}{\mu_{m}} .
$$

The interfacial tension force (4.2) is discretized with the continuum-surface-force model (Brackbill, Kothe \& Zemach 1992). Figure 22 in the Appendix illustrates the marker-and-cell (MAC) grid for the magnetic stress tensor and velocities. The spatial discretization of (4.4) is also defined in the Appendix. In the Navier-Stokes solver, a provisional velocity field is first predicted and then corrected with the pressure field which is calculated as a solution of a Poisson problem (Lafaurie et al. 1994; Li \& Renardy 1999; Scardovelli \& Zaleski 1999; Li, Renardy \& Renardy 2000).

Boundary conditions. A uniform magnetic field $\boldsymbol{H}=\left(0,0, H_{o}\right)$, where $H_{o}$ is the magnetic field intensity at infinity, is imposed at the top and bottom boundaries of the computational domain. In the absence of an interface, $\nabla^{2} \phi=0$ where $\boldsymbol{H}=\nabla \phi$. In cylindrical coordinates with axisymmetry, $\nabla \phi=((\partial \phi / \partial r),(1 / r)(\partial \phi / \partial \theta),(\partial \phi / \partial z))$, and therefore $\partial \phi / \partial r=0, \partial \phi / \partial z=H_{o}$. This yields $\phi(r, z)=H_{o} z+$ constant. Therefore, in order to solve Laplace's equation (2.3) in the presence of an interface, the following boundary conditions are employed: $(\partial / \partial z) \phi=H_{o}$ at $z=0, L_{z}$, and $(\partial / \partial r) \phi=0$ at the side boundary $r=L_{r}$.

Note that a symmetry condition, $(\partial / \partial z) \phi=0$, at $z=0$, and $(\partial / \partial r) \phi=0$, at $r=0$, can be applied. A posteriori checks are conducted to ensure that the distance of the 


$\begin{array}{rc}R_{o} / \Delta & \text { Relative error }(\%) \\ 6.4 & 1.75 \\ 12.8 & 0.71 \\ 25.6 & 0.21 \\ 51.2 & 0.05\end{array}$

TABLE 1. Relative error in computed magnetic field inside the drop as a function of grid refinement. The results converge with mesh refinement.

top and bottom boundaries from the interface is large enough so that the magnetic field remains uniform there.

\subsection{Convergence study}

We now show a test of the accuracy of the calculated magnetic field. A ferrofluid drop of radius $R_{o}=2 \mathrm{~mm}$ is placed at the centre of a non-magnetizable matrix of size $10 R_{o} \times 10 R_{o}$. Because of symmetry only half of the domain is simulated, i.e. $0 \leqslant z \leqslant 10 R_{o}, 0 \leqslant r \leqslant 5 R_{o}$. A uniform magnetic field $\boldsymbol{H}_{o}=\left(0,0, H_{o}\right)$ of intensity $H_{o}=1 \mathrm{kA} \mathrm{m}^{-1}$ is applied at the top and bottom of the boundary. The magnetic susceptibility $\chi$ is set to 1 . The surface tension is not incorporated to ensure that corresponding errors are not due to the discretization of the surface tension. We perform the simulations for only one time step.

For an undeformed ferrofluid drop under a uniform magnetic field, the analytical solution for the magnetic field interior of the spherical drop was given in $\S 3.1$ :

$$
H_{\text {in }}=\frac{3}{3+\chi} H_{o},
$$

which is the well-known Clausius-Mossotti formula (Markov 1999; Mikkelsen 2005). Table 1 presents the convergence of the difference between the calculated magnetic field inside the drop and the theoretical value. We define the error in the $L_{1}$ norm by $\sum_{i=1}^{N}\left|H_{i, \text { exact }}-H_{i, \text { computed }}\right| \Delta^{2}$, where the sum is over cells that are completely filled with the ferrofluid and $N$ is the number of cells. Table 1 shows that the relative errors in computed magnetic field inside the drop converge to zero with refinements in mesh size $\Delta$. Figure $5(a)$ shows the contours of the magnetic field on the left and magnetic field lines on the right. It is evident that the magnetic field inside the drop remains uniform, collinear with the applied magnetic field, and is a factor $3 /(3+\chi)$ of the applied field. The magnetic field in the non-magnetizable surrounding medium is distorted in the proximity of the drop and reaches the uniform magnetic field $\boldsymbol{H}_{o}$ away from the drop. Figure 5(b) presents the magnetic field potential; the potential lines are uniform inside the drop and at boundaries, but non-uniform in the nonmagnetizable medium in the presence of the ferrofluid drop because of the jump in permeability across the interface.

The numerical validation for the pressure jump across interface is given in figure 6 . The analytical prediction obtained by the balance of stresses along the direction normal to the interface for a spherical ferrofluid drop is given in (3.5) in $\S 3.1$. The computed pressure jump is obtained across the interface from the pressure distribution along $\theta=0,45$ and $90^{\circ}$. The pointwise error is defined by $\left|\llbracket p \rrbracket_{\text {exact }}-\llbracket p \rrbracket_{\text {computed }}\right| /\left|\llbracket p \rrbracket_{\text {exact }}\right|$. Figure 6 shows the decrease in error in the numerically computed pressure jump at the interface with grid refinement. 

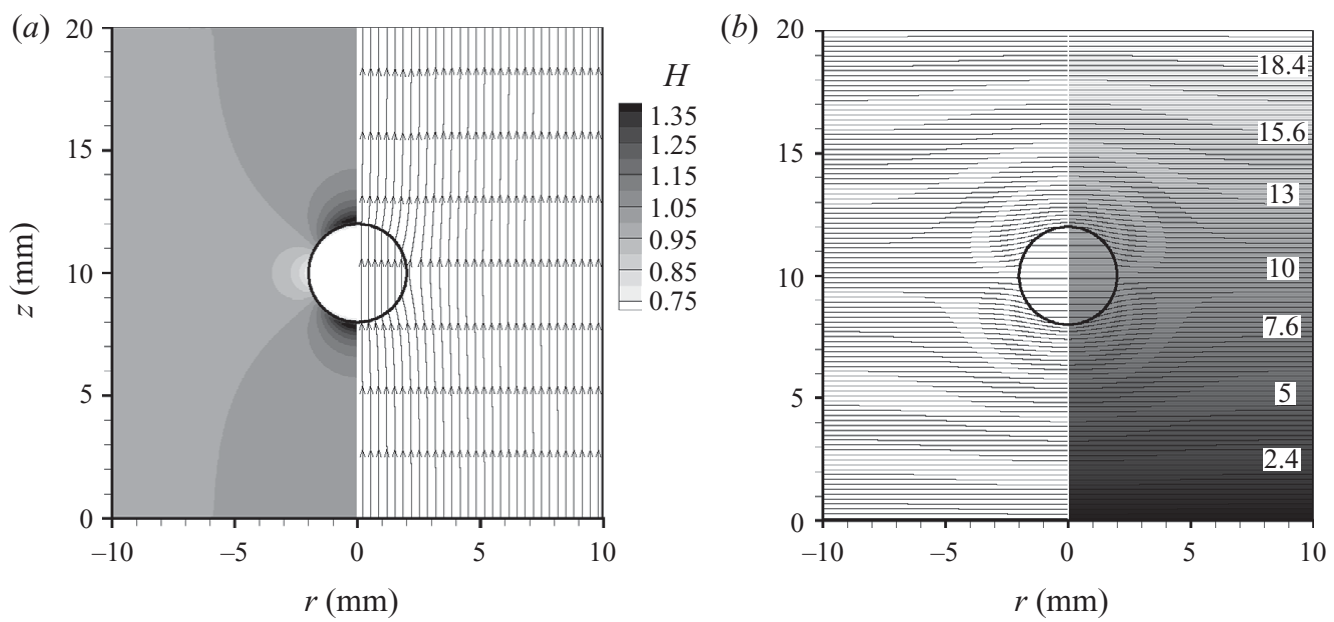

FigurE 5. (a) Contours of the magnetic field $\left(\mathrm{kA} \mathrm{m}^{-1}\right)$ on the left and magnetic field lines on the right and $(b)$ magnetic field potential (A) are plotted in the presence of a ferrofluid droplet in a non-magnetizable medium. $\chi=1$.

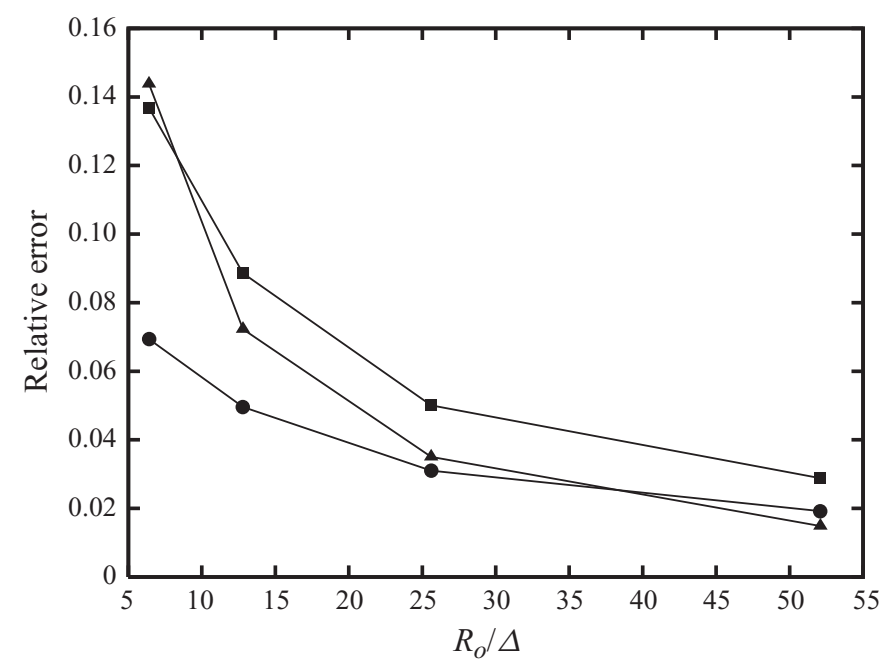

FIGURE 6. Relative error, $\left|\llbracket p \rrbracket_{\text {exact }}-\llbracket p \rrbracket_{\text {computed }}\right| /\left|\llbracket p \rrbracket_{\text {exact }}\right|$, in the calculated pressure jump at $\theta=90^{\circ}(\mathbf{\Delta}), 45^{\circ}(\bullet)$ and $0^{\circ}(\mathbf{\square})$ as a function of spatial refinement.

\subsection{Drop at equilibrium under a uniform magnetic field}

The theoretical analysis for a prolate spheroid drop in $\S 3.2$ yields a relationship (3.16) between the equilibrium drop aspect ratio and the magnetic Bond number (2.8) (cf. figure 3). In this section, transient direct numerical simulations to stationary states are performed without the assumption of the spheroidal shape. We choose $R_{o}=1 \mathrm{~mm}$, interfacial tension $\sigma=1 \mathrm{mN} \mathrm{m}^{-1}$ and a constant $\mu_{d}$, which is independent of the magnetic field for the linearly magnetizable ferrofluid. Note that the results are checked to be independent of the size of the computational box, mesh size, time step and the small transient Reynolds number Re. 


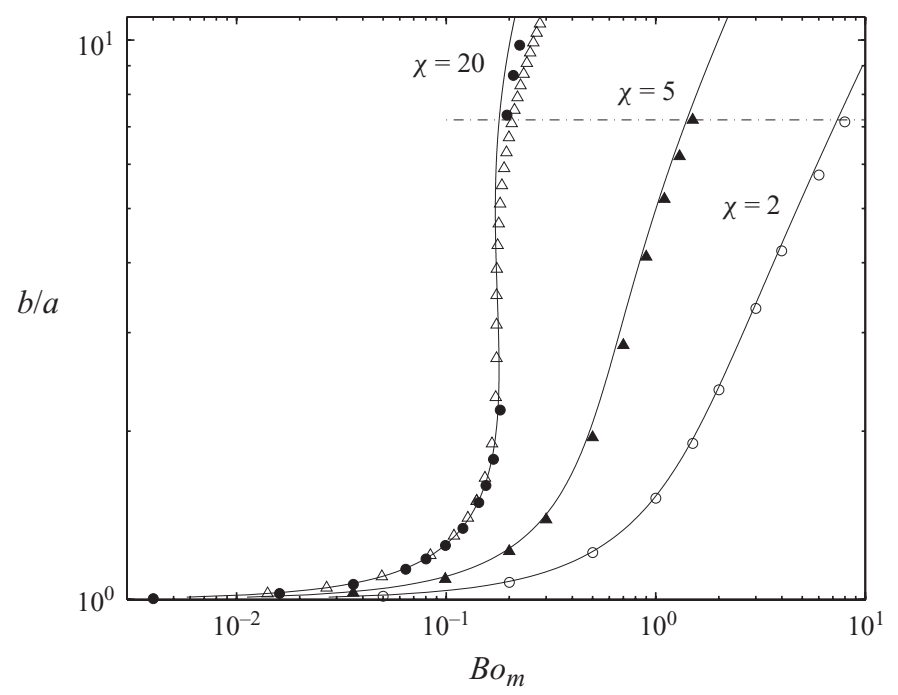

FIGURE 7. Comparison of the dependence of the drop aspect ratio $b / a$ on the magnetic Bond number $\mathrm{Bo}_{m}$. Solid lines represent the theory of $\S 3.2$. Numerical results are presented for $\chi=2$ $(\bigcirc), \chi=5(\Delta)$ and $\chi=20(\bullet)$. The prediction of Bacri \& Salin (1982) is denoted by $\triangle$. The shapes for $\chi=20,5$ and 2 at the same aspect ratio 7 (-.-) are examined further in figure 9 .

Figure 7 shows that excellent agreement is achieved between the numerical and theoretical studies. The solid lines represent (3.16), the symbol $(\triangle)$ is the prediction of Bacri \& Salin (1982) and the numerical results are given at $\chi=2(0), \chi=5(\boldsymbol{\Delta})$ and $\chi=20(\bullet)$. From the computational results alone, it is observed that at sufficiently large $\chi$, the drop equilibrium aspect ratio jumps to a higher value when the magnetic Bond number reaches a critical value, while for small values of $\chi$, the drop equilibrium aspect ratio increases continuously as a function of the magnetic Bond number, in agreement with the theories. Specifically, at $\chi=20$, the jump in drop shape occurs when the magnetic Bond number changes from $B o_{m}=0.18$ to 0.19 . Figure 7 also confirms that at a fixed Bond number, the equilibrium aspect ratio increases with the magnetic susceptibility $\chi$.

Figure 8 shows the computed drop equilibrium shapes at $\chi=20$ from the spheroid at $B o_{m}=0.1$, through the gradually elongating prolate shapes at $B o_{m}=0.14,0.17,0.18$, and finally the highly elongated shape with nearly conical ends at $B o_{m}=0.19$. Therefore, the critical magnetic Bond number lies between 0.18 and 0.19. This shape transition is also observed experimentally at high $\chi$ by Bacri \& Salin (1982). Note also that at $\chi=20$, the shape produced at $B o_{m} \geqslant 0.19$ remains stable, as in the experimental work of Bacri \& Salin (1982). When the Bond number increases past the critical value, it is observed numerically that the width of the nearly conical tips decreases.

We find that for a fixed $b / a \geqslant$ roughly 7 (see the dash-dotted line in figure 7), the shape at $\chi=20$ has conical ends, while the ends remain rounded at the lower values of $\chi=5$ and 2, which suggests that there is a relationship between the magnetic susceptibility $\chi$, the aspect ratio $b / a$ and the magnetic Bond number $B o_{m}$ at which conical ends are first observed. Figure 9 shows the equilibrium shapes at $(a) \chi=20$, (b) $\chi=5$ and (c) $\chi=2$ for $b / a=7$ (dash-dotted line in figure 7). These observations are consistent with experimental measurements performed by Bacri \& Salin (1982). 


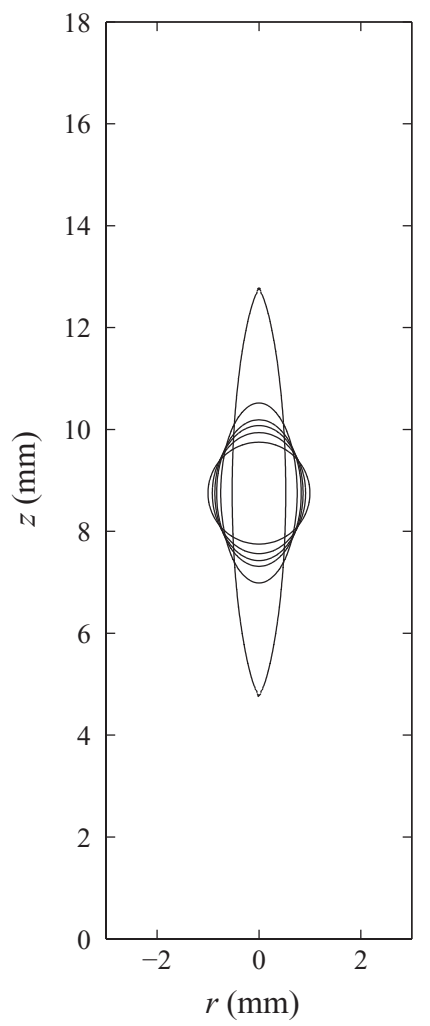

FIGURE 8. Interface equilibrium configurations at $\chi=20$ for $B o_{m}=0.1,0.14,0.17,0.18,0.19$. A slight variation in $B o_{m}=0.18$ to 0.19 results in the transition of the drop shape from rounded to nearly pointed ends.
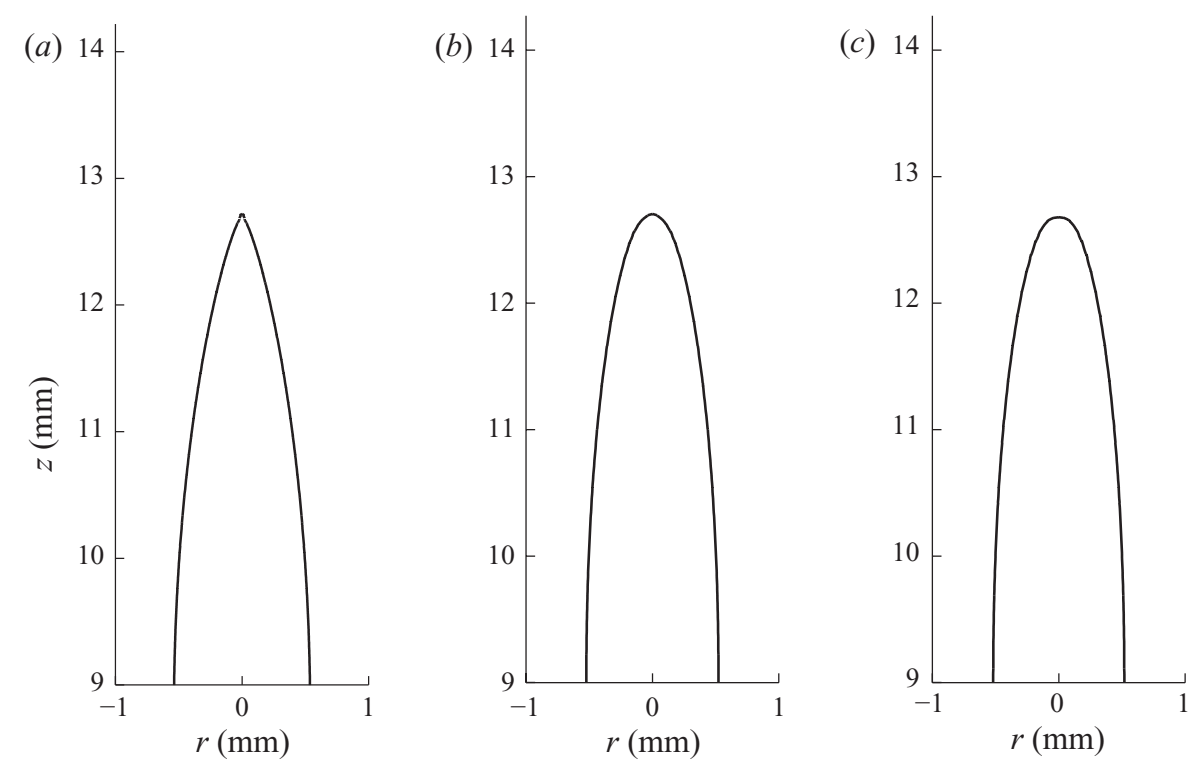

FIGURE 9. Drop half-shapes for varying $\chi,(a) \chi=20,(b) \chi=5$ and $(c) \chi=2$, at the drop equilibrium aspect ratio $b / a=7$ (-.-, figure 7). Nearly conical tips are observed at $\chi=20$. 


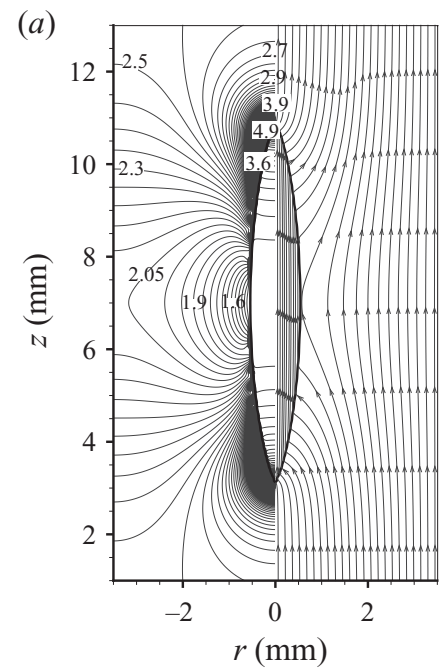

(b)

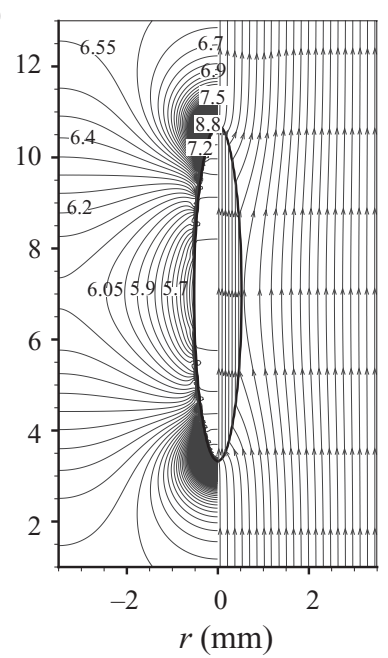

(c)

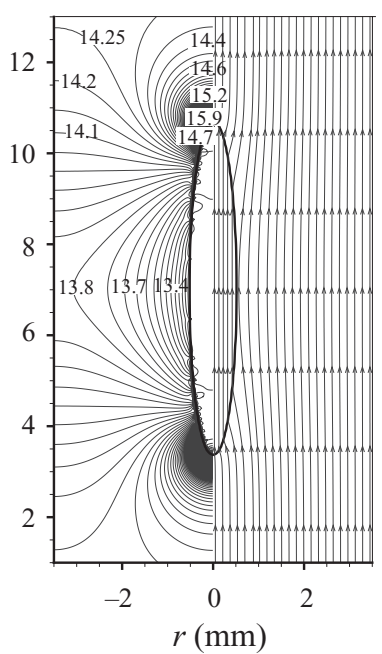

FIGURE 10. Contours of the magnetic field amplitude $\left(\mathrm{kA} \mathrm{m}^{-1}\right.$, left $)$ and magnetic field lines (right) for the highly deformed drop suspended in a non-magnetizable medium of figure 9; $b / a=7$. (a) $\chi=20, B o_{m}=0.2$, (b) $\chi=5, B o_{m}=1.5$ and $(c) \chi=2, B o_{m}=8$.

The simulated magnetic fields which correspond to the drop shapes in figure 9 for $b / a=7$ are illustrated in figure 10 . The magnetic field lines inside each highly deformed drop as well as in the non-magnetizable surrounding medium are shown at (a) $\chi=20, B o_{m}=0.2 ;(b) \chi=5, B o_{m}=1.5$; and (c) $\chi=2, B o_{m}=8$. We note that the effect of the tangential component of the magnetic field which is responsible for the appearance of conical ends is more prominent at $\chi=20$ (figure 10a), while at $\chi=2$ (figure 10c) the effect of the normal component of the magnetic field which is responsible for the appearance of rounded ends is more visible.

It is evident from figure 7 that for permeability ratios larger than 20 and for Bond numbers much less than 0.1, the drop aspect ratio is a multi-valued function of the Bond number. A solution with high elongation and conical tips appears. Our numerical methodology can handle such a case as long as a more refined mesh and a larger computational domain is used than that shown in figure 8 .

\section{Results: comparison with experimental measurements}

In this section, we return to the experimental results exemplified in figure 2 and taken with the instrument shown in figure 11. The density of the ferrofluid drop is matched with the surrounding glycerol medium at $1260 \mathrm{~kg} \mathrm{~m}^{-3}$. The value of the interfacial tension for the fluid pair is not readily available; because of the high viscosity of the ferrofluid, direct measurements are difficult to do. It is therefore found from a comparison of numerically simulated shapes with experimental data at the low field. Two series of experiments are performed. For low field data, the magnetic field is varied from 0.397 to $6.931 \mathrm{kA} \mathrm{m}^{-1}$. For high field data, the magnetic field is varied from 8.093 to $837.950 \mathrm{kA} \mathrm{m}^{-1}$.

A series of experiments are conducted to understand the behaviour of the PDMS ferrofluid in uniform magnetic fields. Figure 12 shows photographs of the drop at equilibrium for the magnetic field strengths $H_{o}=6.032,12.167,23.947,59.762$, $162.33 \mathrm{kA} \mathrm{m}^{-1}$. We compare the experimental data with direct numerical simulations. 

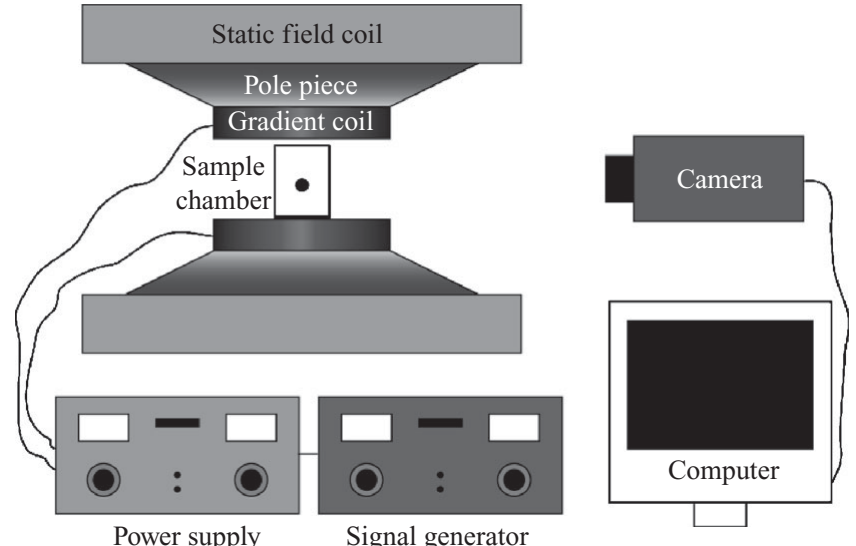

FIGURE 11. Instrument schematic for the experimental data on deformation of a PDMS ferrofluid drop in a viscous medium under applied uniform magnetic fields $\boldsymbol{H}=\left(0,0, H_{o}\right)$, presented in $\S 5$.

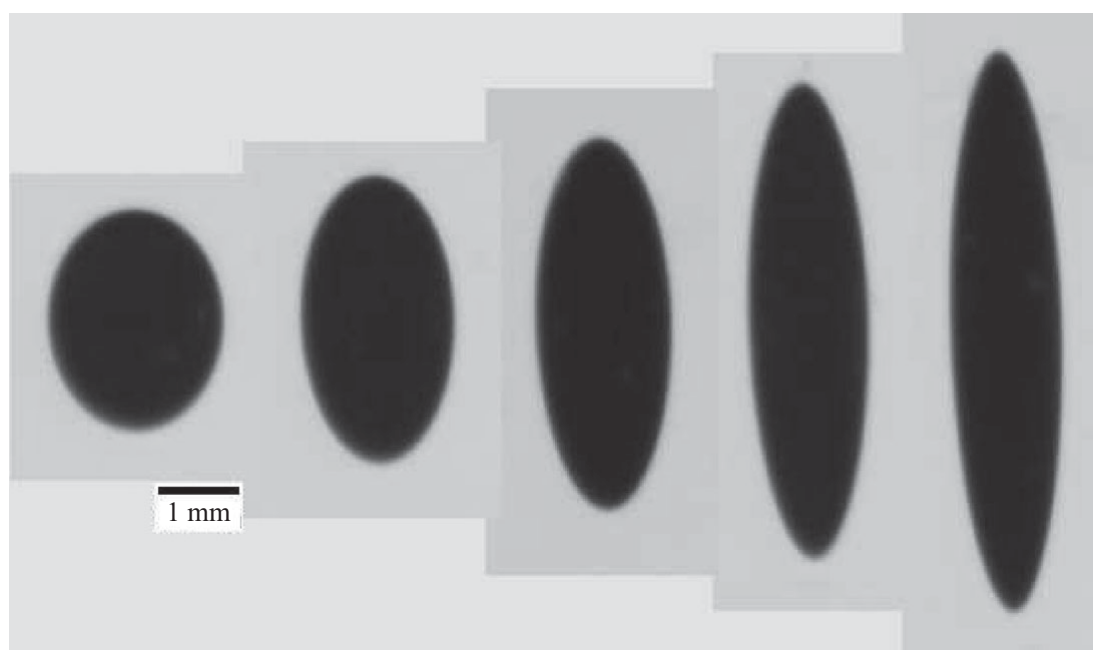

FIGURE 12. Photographic images of the drop equilibrium shape taken at magnetic field strengths $H_{o}=6.032,12.167,23.947,59.762,162.33 \mathrm{kA} \mathrm{m}^{-1}$ (from left to right).

The magnetization of the PDMS ferrofluid as a function of the internal field was measured in a 7 Tesla Quantum Design MPMS SQUID magnetometer at room temperature. Figure 13 shows the $\boldsymbol{M}$ versus $\boldsymbol{H}$ relationship measured in the SQUID magnetometer and a fit of the Langevin function to the experimental data with the saturation magnetization $M_{s} \approx 24 \mathrm{kA} \mathrm{m}^{-1}$ and $m \approx 1.8 \times 10^{-19} \mathrm{~A} \mathrm{~m}^{2}$. Because of the particle-size distribution, the fit is not perfect. However, it is possible to improve the quality of the fit by integrating the Langevin function over a particle-size distribution (Woodward et al. 2007). While the low field data show that the magnetization is a linear function of the applied internal magnetic field (see $\S 5.1$ ), at high internal field, the magnetization of the ferrofluid saturates, consistent with the superparamagnetic behaviour of the magnetic particles in the ferrofluid (see also $\S 5.2$ ). 


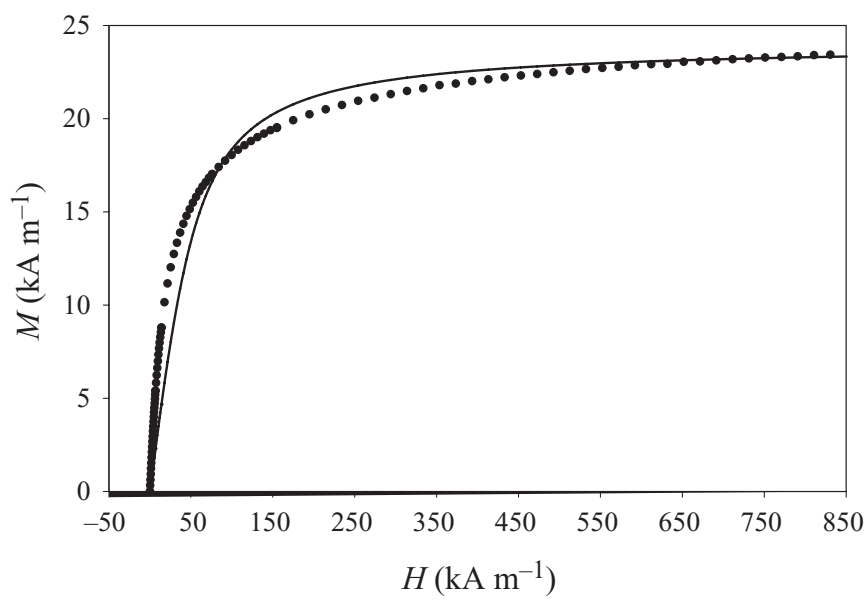

FIGURE 13. Magnetization behaviour of the ferrofluid containing 7 vol. \% of magnetite $\left(\mathrm{Fe}_{3} \mathrm{O}_{4}\right)$ particles with a mean diameter of $7.2 \mathrm{~nm}$. Measured data $(\bullet)$ are compared with the Langevin fit $\left(-\frac{-}{-}\right.$ ), assuming each of the magnetite particles has a total magnetic moment $m \approx 1.8 \times 10^{-19} \mathrm{~A} \mathrm{~m}^{2}$.

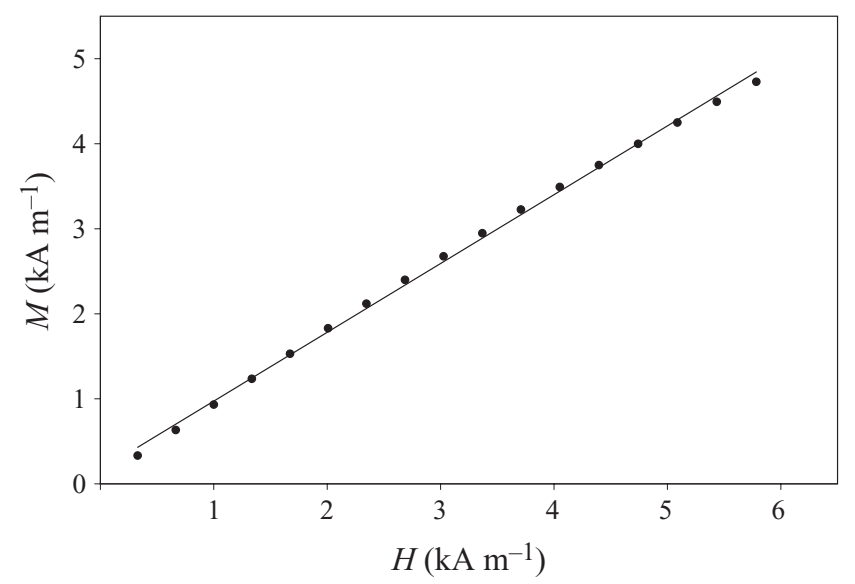

FIGURE 14. Low field magnetization of the ferrofluid as a function of the internal magnetic field. A linear fit, namely $M=0.8903 H+0.1634$, describes the actual magnetization versus the internal magnetic field, measured by the SQUID magnetometer $(\bullet)$.

\subsection{Numerical simulation for the low field data}

Direct numerical simulations are conducted for a ferrofluid drop of radius $R_{o}=1.291 \mathrm{~mm}$, positioned at the centre of a computational domain of size $L_{z}=15 \mathrm{~mm}$ by $L_{r}=7.5 \mathrm{~mm}$. The computational domain is checked to be sufficiently large that results do not change if a larger size is used. The mesh size is $\Delta=R_{o} / 16.5$ and the time step $\Delta t=10^{-5} \mathrm{~s}$. The viscosity of both fluids is chosen to be $0.1 \mathrm{~Pa}$ s. This does not correspond to the experimental situation; however, we are interested only in the final shape, which does not depend on the viscosity, and we have therefore chosen a value which optimizes the computational time for the transition. Figure 14 presents the magnetization $M$ of the ferrofluid at low fields $\left(0-6.0 \mathrm{kA} \mathrm{m}^{-1}\right)$. We note that the magnetization increases linearly with the magnetic field and, consequently, a constant value for the magnetic susceptibility $\chi=0.8903$ is found. However, for the 


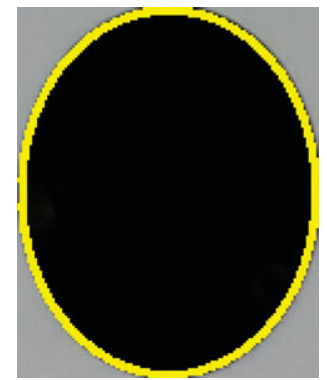

FIGURE 15. (Colour online) The numerically simulated equilibrium shape is highlighted and superimposed over the experimentally visualized shape of the drop for an applied uniform magnetic field of magnitude $H_{o}=5.96 \mathrm{kA} \mathrm{m}^{-1}$.

numerical simulations, values of magnetic susceptibility are computed for each value of the magnetic field from the subsequent experimental measurement. This turns out to result in an improved comparison with measured values.

We first examine the experimental measurements at $H_{o}=5.96 \mathrm{kA} \mathrm{m}^{-1}$. These moderate field data are chosen to avoid any measurement errors that may exist in very low field data. We find that this results in subsequent numerical simulations that agree with experimentally measured elongations over a wider range of applied magnetic fields. We perform numerical simulations with a variety of values for the surface tension coefficient with other parameters fixed, until the numerically simulated drop matches the experimental visualization. This yields the value of the interfacial tension $\sigma=13.5 \mathrm{~m} \mathrm{~N} \mathrm{~m}^{-1}$. Figure 15 shows the calculated equilibrium configuration (highlighted) superimposed over the experimentally visualized drop (dark). This value for the interfacial tension is then used to numerically simulate the experimental data for magnetic fields ranging from 0.397 to $6.931 \mathrm{kA} \mathrm{m}^{-1}$.

Figure 16 presents the comparison of direct numerical simulations with experimental data, as well as with the theoretical prediction based on (3.16). We note that for the theoretical fit, a constant value of magnetic susceptibility $\chi=0.8903$ is used. This value is found from the linear fit of the experimentally measured magnetization versus internal field. The agreement between the numerical result and the analytical study is excellent. Moreover, the numerical simulations predict the experimental data very well in moderate magnetic fields. However, at applied magnetic fields lower than $H_{o}=3 \mathrm{kA} \mathrm{m}^{-1}$, the predicted aspect ratio is smaller than the experimental data due to subsequent increase of measurement errors in experimental data. Figure 17 shows the drop shapes which correspond to figure 16 . The numerically simulated drop shapes are highlighted and superimposed over the dark experimentally visualized images at applied magnetic field strengths $H_{o}=3.580,3.978,4.774,5.172,6.366 \mathrm{kA} \mathrm{m}^{-1}$. Note that for this range of magnetic field, figure $17(a-e)$ clearly shows that the numerical simulations predict the experimentally observed drop configurations very well.

\subsection{Simulation for the high field data}

In order to simulate the high field response, which has a larger aspect ratio, the computational domain of $\S 5.1$ is increased to $L_{z}=22.5 \mathrm{~mm}$ by $L_{r}=7.5 \mathrm{~mm}$, with mesh size $\Delta=R_{o} / 22$. In high magnetic fields $\left(8-80 \mathrm{kA} \mathrm{m}^{-1}\right)$ the permeability of the ferrofluid is seen to vary with the applied field and takes a shape typical for an ensemble of superparamagnetic particles. Therefore, an effective magnetic susceptibility that is a function of the magnetic field and relates the magnetization to the applied magnetic field must be used. Here we use a logarithmic function to describe the behaviour of 


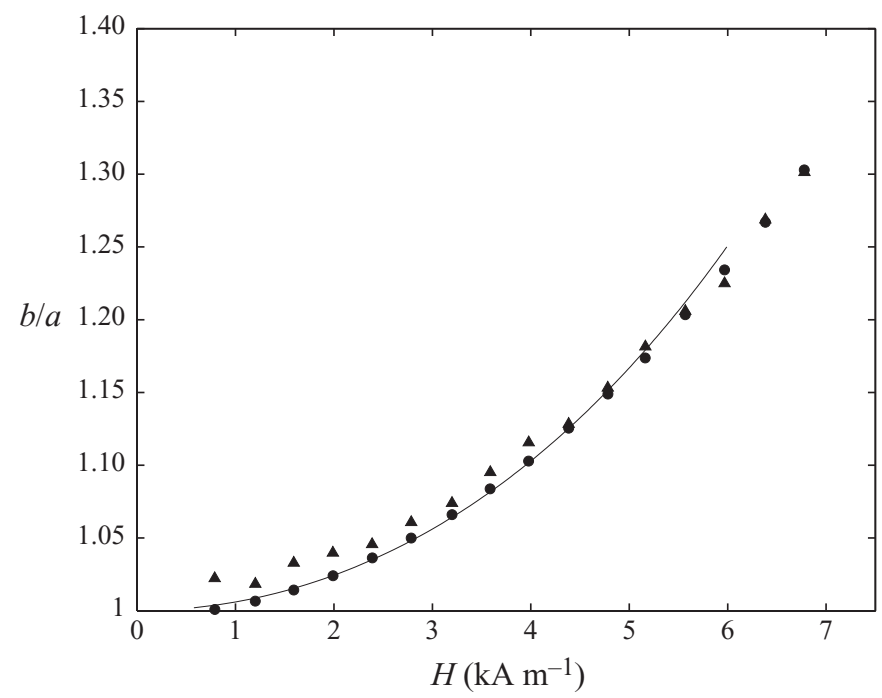

FIGURE 16. Comparison of direct numerical simulations $(\bullet)$ with experimental data $(\boldsymbol{\Delta})$ and with the theory discussed in $\S 3.2(-)$ for low applied fields. Drop shapes are shown in figure 17.

(a)

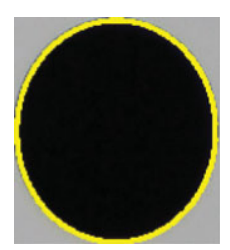

(b)

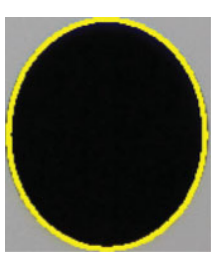

(c)

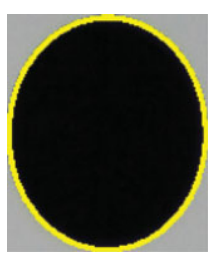

$(d)$

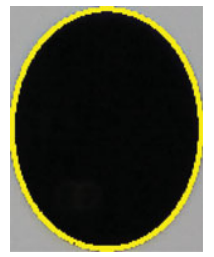

(e)

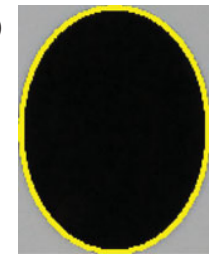

FIGURE 17. (Colour online) Numerically simulated drop equilibrium shapes are highlighted and superimposed over experimentally obtained images. The values for the applied magnetic field $\left(0,0, H_{o}\right) \mathrm{kA} \mathrm{m}^{-1}$ are $H_{o}=(a) 3.978,(b) 4.774,(c) 5.172,(d) 6.366$ and $(e) 6.764$.

the magnetization of the ferrofluid versus the applied magnetic field. Figure 18 shows the high-field magnetization of the ferrofluid measured by the SQUID magnetometer fitted with a logarithmic function. As shown, the fit is perfect, which is essential for the accurate computations of high field numerical simulations.

Figure 19 presents the direct numerical simulations conducted at magnetic fields ranging from 7.989 to $79.975 \mathrm{kA} \mathrm{m}^{-1}$, using the value for interfacial tension determined at $5.96 \mathrm{kA} \mathrm{m}^{-1}\left(13.5 \mathrm{mN} \mathrm{m}^{-1}\right)$. While the comparison of numerical simulations and the data at $H_{o}=7.989 \mathrm{kA} \mathrm{m}^{-1}$ is reasonable, at fields above $12.167 \mathrm{kA} \mathrm{m}^{-1}$, the deviations between the numerical and experimental results become apparent. One can represent this effect in terms of a variation in the apparent interfacial tension with applied magnetic field. We explore this numerically, since conventional experimental measurements of surface tension in magnetic fields are not possible. We computationally determine the surface tension at a given applied magnetic field by comparing the simulated shapes with experimental data until a match is obtained. Figure 20 shows the numerically simulated surface tension coefficient as a function of the magnetic field strength. The solid line is provided as a guide to the eye. The figure shows that the surface tension increases as the magnetic field increases and approaches a limiting value. The values noted on the curve denote the numerically 


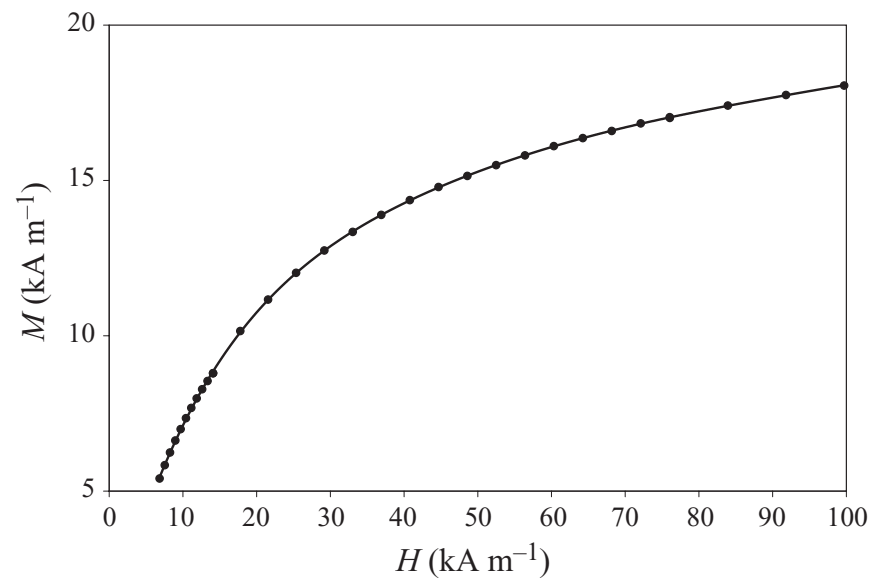

FIGURE 18. Magnetization behaviour of the ferrofluid for fields varying from 8 to $80 \mathrm{kA} \mathrm{m}^{-1}$ fitted with a logarithmic function $M=4.8568 \ln (H)-3.956$.

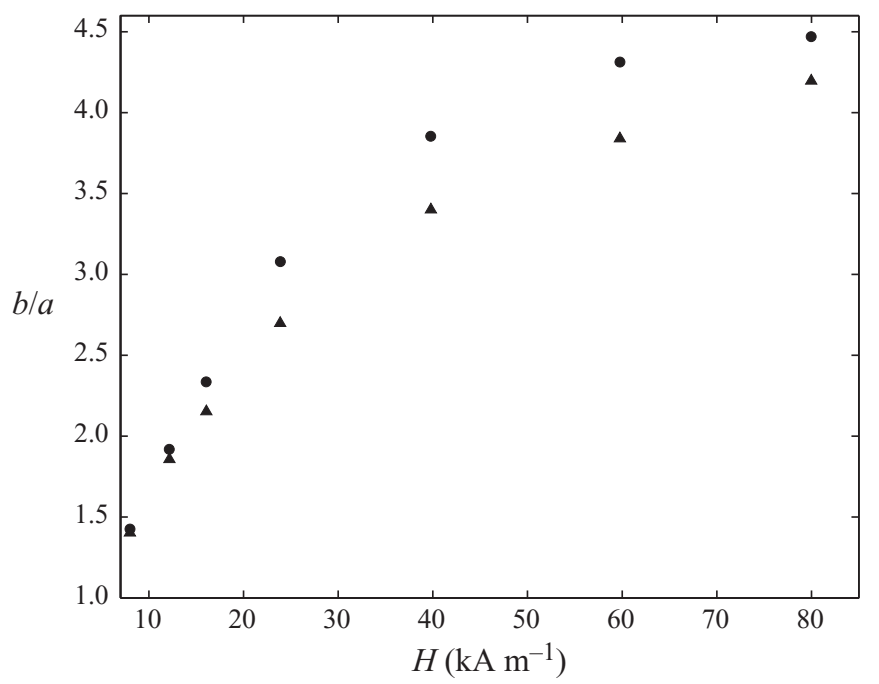

Figure 19. Direct numerical simulations $(\bullet)$ and experimental data $(\mathbf{\Delta})$. At high magnetic field strengths, numerical simulations begin to deviate from experimental data.

simulated drop aspect ratios $b / a$, which are equal to the experimentally observed values. Figure 21 shows numerically simulated configurations superimposed over photographic images at $H_{o}=(a)$ 7.989, (b) 12.167, (c) 16.074, (d) 23.873, (e) 39.947 and $(f) 59.76 \mathrm{kA} \mathrm{m}^{-1}$ for the surface tensions given in figure 20 . Given the variation in the computed surface tension over the field range from 0.397 to $79.975 \mathrm{kA} \mathrm{m}^{-1}$, it is clear that no single value for the surface tension will provide an accurate numerical simulation of all of the observed droplet shapes. At moderate fields the apparent surface tension is relatively constant. At fields of above $7 \mathrm{kA} \mathrm{m}^{-1}$, there are significant changes in the computed surface tension, which explains the discrepancy between the numerical and experimental results in figure 19. At high fields, the computed surface tension increases rapidly and then appears to saturate beyond fields around $16 \mathrm{kA} \mathrm{m}^{-1}$. This saturation occurs at fields that are much smaller than the fields at which the magnetization saturates. 


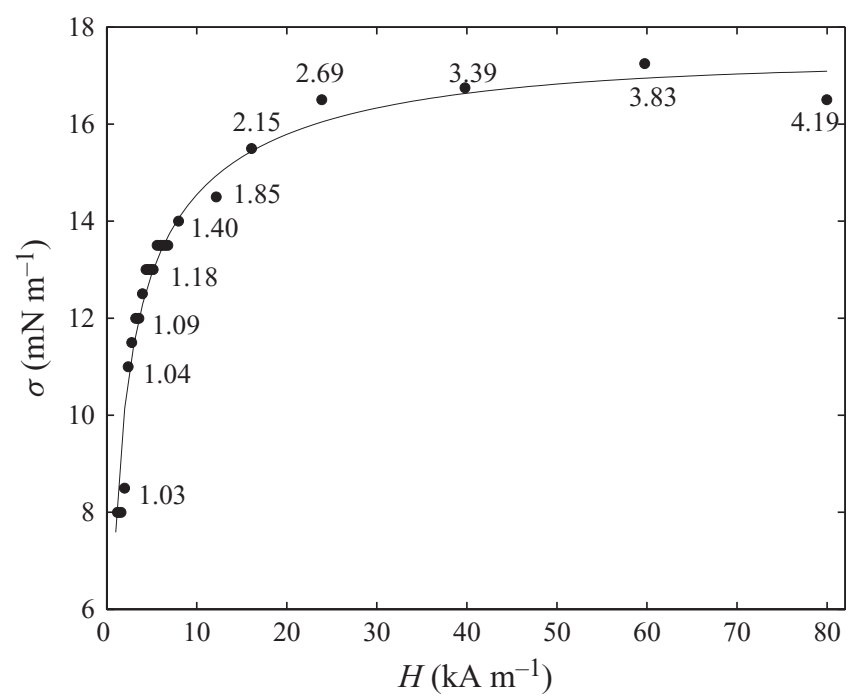

FigURE 20. Surface tension versus applied field strength. Values of the drop aspect ratio $b / a$ are noted along the line. The solid line is a guide for the eye and represents $\sigma=-0.4107 \ln (H)^{2}+3.968 \ln (H)+7.587$. The drop shapes are shown in figure 21 .

(a)

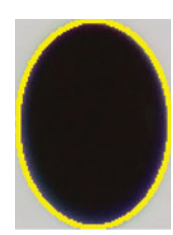

(b)

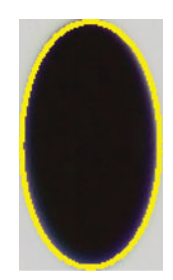

(c)

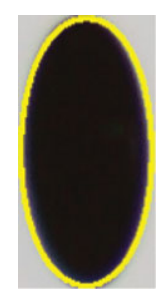

(d)

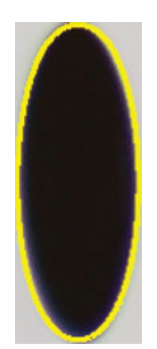

(e)

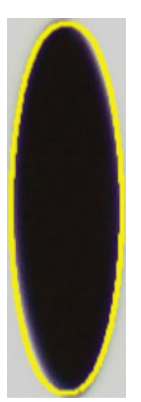

$(f)$

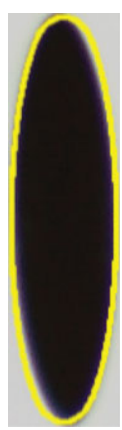

FIGURE 21. (Colour online) The numerically simulated equilibrium shapes are highlighted and superimposed over the experimentally obtained dark images at $H_{o}=(a) 7.989,(b) 12.167$, (c) $16.074,(d) 23.873,(e) 39.947$ and $(f) 59.76 \mathrm{kA} \mathrm{m}^{-1}$, for surface tension varying as a function of the imposed field. Figure 20 shows the data points with drop aspect ratios $b / a$ given along the curve.

\section{Conclusion}

The effect of applied magnetic fields on the deformation of a bio-compatible hydrophobic ferrofluid drop suspended in a viscous medium is investigated numerically and compared with experimental data. Analytical formulae for ellipsoidal drops and near-spheroidal drops are reviewed and developed for code validation. Numerical validation tests show good agreement with theoretical predictions. At low magnetic fields, both the experimental and numerical results follow the asymptotic small deformation theory. The advantage of the numerical simulations over the analytical solutions is that the numerical simulations can fit data where the assumption of prescribed spheroidal or ellipsoidal droplet shapes in the analytical solutions is no longer valid. 
Computed drop shapes deviate from experimental results with increase in magnetic field strength when surface tension is assumed to be a constant, which is determined from low field data. This behaviour has been represented here in terms of dependence of apparent interfacial tension on the magnetic field. This idea is investigated computationally by varying the surface tension as a function of the applied magnetic field and by comparing the simulated drop shapes with experimental measurements until matched. In the numerical simulations, we have varied the parameters to examine sensitivity. For instance, the permeability of the PDMS ferrofluid was varied to examine the sensitivity of the results. We found that the results are not sensitive to small changes (less than $5 \%$ ) of the PDMS susceptibility and also the change in the surface tension coefficient (less than $5 \%$ ) made a small difference in drop deformation.

The possibility that the deviations between the experimental and computational results are temperature-related has been considered, but there is insufficient field cycling to generate an appreciable heating of the ferrofluid itself. In addition, we have tested that there is no heating from external sources such as the electromagnets or gradient field coils. We might consider the possibility of a magnetorheological effect. Viscosity measurements on the ferrofluid used in this study suggest a yield stress (Mefford 2007) and it is generally known that the yield stress in magnetic fluids can increase in a magnetic field (Shahnazian \& Odenbach 2007). If the drop stops deforming before equilibrium of stresses is reached, this might also explain why the drops in the experiments deform less at a field than theory would lead us to expect. However, the drop shape changes rapidly on the application of a rapid magnetic field change, even in the range where the apparent interfacial tension appears to saturate.

The difference between the experimental and computational results that is represented here as a variation in apparent interfacial energy must then be explained by either a decrease in the magnetic energy or an increase in the surface energy of the droplet. One way in which magnetic fields affect surface tension is through dipole interactions (Cebers 1982, 2002; Liggieri, Sanfeld \& Steinchen 1994) within the fluid. However, this effect is orders of magnitude smaller than the change in surface tension observed here and hence is unlikely to be the driving force for the observations reported here.

One of the critical assumptions in both the analytical and computational models is that the ferrofluid can be treated as a uniform material with uniform magnetic properties. However, a ferrofluid is an inhomogeneous composite containing magnetic particles, and non-magnetic surfactants and carrier fluids. We therefore postulate how changes in such non-uniform materials might affect the magnetic and surface energies. When fields are applied to such ferrofluids, various forms of ordering occur within them, such as chain formation (Mendelev \& Ivanov 2004) and phase separation. The formation of chains has been shown to lead to an increase in the bulk susceptibility of a ferrofluid due to enhanced dipole-dipole interactions between particles (Martin, Venturini \& Huber 2008). However, such changes in susceptibility would have also occurred during the SQUID magnetometer measurements and hence would be taken into account in the modelling. Furthermore, such changes would lead to an increase rather than a decrease in the magnetic energy. Another possibility is that application of a magnetic field may cause spatial fractionation of the magnetic nanoparticles based on variations in their magnetic properties such as magnetic susceptibility. We could speculate, for instance, that an applied field results in a spatial variation of magnetic susceptibility within the drop, inducing a non-uniform magnetic state that leads to an effective lowering of the magnetostatic energy of the drop. 
An alternative mechanism for the observations could be an increase in surface roughness related to phenomena such as chain formation within the droplet (Mefford 2007; Mefford et al. 2008b). Such a surface roughness could increase the surface area. However, there is no evidence for surface roughness from the high-resolution imaging of the droplets. Therefore, any such roughness would have to have a length scale of less than $20 \mu \mathrm{m}$. Further experiments with higher spatial resolution will be required to investigate this possibility.

Experiments were also done on more conventional ferrofluids that have a carrier fluid (Tyler, Woodward \& St. Pierre 2010). These fluids are much less viscous than the fluid considered in this paper, and thus independent measurements of surface tension are much easier to do. The results suggest that the low field data are consistent with the surface tension measured in the absence of a field, while the high field data are closer to the surface tension of the carrier fluid without magnetic particles. This points to the possibility that the particles act as a surfactant, but as a field is applied, particles retreat from the surface or at least parts of the surface, thereby leading to the increase in surface tension. Given these results, we conclude that the change in apparent surface tension is probably dominated by a change in surface physicochemical properties associated with the inhomogeneous nature of the ferrofluid and rearrangement of nanoparticles at the surface.

This material is based in part upon work supported by the National Science Foundation under grants DMS-0456086, DMS-0907788 and DMS-0707727, the Institute for Mathematics and its Applications (Minnesota) during the autumn of 2009, the TeraGrid Large Resource Allocations Grant MCA08X019, NCSA grant CTS060022, the Commonwealth of Australia under the International Science Linkages program, ARC Nanotechnology Network and the University of Western Australia Convocation Postgraduate Travel Award. We thank A. Avila for the use of the CEMCC, Universidad de La Frontera, SGI system. We thank O. T. Mefford and the referees for their constructive comments.

\section{Appendix. Spatial discretization}

In axisymmetric coordinates $(r, z, \theta)$, the magnetic field is given by

$$
\boldsymbol{H}=\left(\frac{\partial \phi}{\partial r}, \frac{\partial \phi}{\partial z}, 0\right)
$$

and the corresponding Maxwell stress is

$$
\boldsymbol{\tau}_{m}=\mu\left[\begin{array}{ccc}
\phi_{r}^{2}-\frac{1}{2}\left(\phi_{r}^{2}+\phi_{z}^{2}\right) & \phi_{r} \phi_{z} & 0 \\
\phi_{r} \phi_{z} & \phi_{z}^{2}-\frac{1}{2}\left(\phi_{r}^{2}+\phi_{z}^{2}\right) & 0 \\
0 & 0 & -\frac{1}{2}\left(\phi_{r}^{2}+\phi_{z}^{2}\right)
\end{array}\right] .
$$

We have

$$
\nabla \cdot \boldsymbol{\tau}_{m}=\left[\frac{1}{r} \frac{\partial}{\partial r}\left[r\left(\boldsymbol{\tau}_{m}\right)_{r r}\right]+\frac{\partial}{\partial z}\left[\left(\boldsymbol{\tau}_{m}\right)_{r z}\right]\right] \boldsymbol{e}_{r}+\left[\frac{1}{r} \frac{\partial}{\partial r}\left[r\left(\boldsymbol{\tau}_{m}\right)_{r z}\right]+\frac{\partial}{\partial z}\left[\left(\boldsymbol{\tau}_{m}\right)_{z z}\right]\right] \boldsymbol{e}_{z},
$$

where $\boldsymbol{e}_{r}$ and $\boldsymbol{e}_{z}$ are unit vectorsin the $r$ - and $z$-directions. The magnetic potential field 


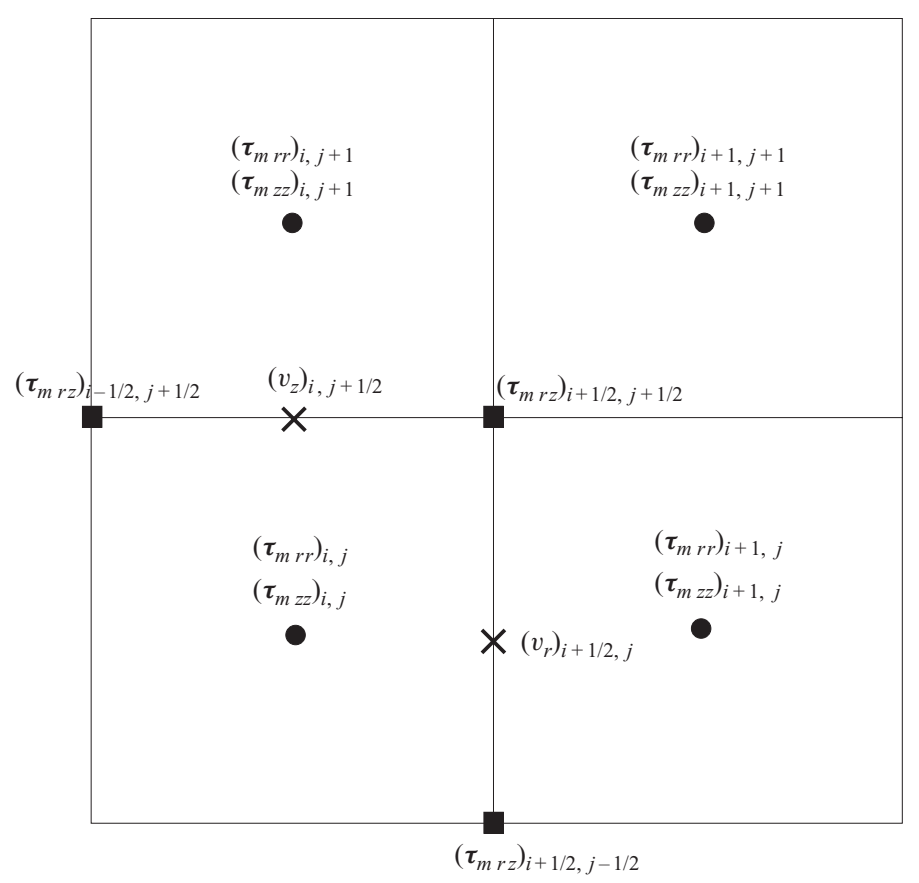

FIGURE 22. Location of the velocities and the magnetic stress tensor components on a MAC grid.

is discretized using second-order central differences. In axisymmetric coordinates, the discretization of $(4.4)$ at cell $(i, j)$ yields

$$
\begin{gathered}
\nabla \cdot(\mu \nabla \phi)_{i, j}=\frac{1}{r_{i, j}} \frac{r_{i+1 / 2, j} \mu_{i+1 / 2, j}\left(\frac{\partial \phi}{\partial r}\right)_{i+1 / 2, j}-r_{i-1 / 2, j} \mu_{i-1 / 2, j}\left(\frac{\partial \phi}{\partial r}\right)_{i-1 / 2, j}}{\Delta} \\
+\frac{\mu_{i, j+1 / 2}\left(\frac{\partial \phi}{\partial z}\right)_{i, j+1 / 2}-\mu_{i, j-1 / 2}\left(\frac{\partial \phi}{\partial z}\right)_{i, j-1 / 2}}{\Delta}, \quad
\end{gathered}
$$

where, for instance for the cell face $(i+1 / 2, j)$,

$$
\left(\frac{\partial \phi}{\partial r}\right)_{i+1 / 2, j}=\frac{\phi_{i+1, j}-\phi_{i, j}}{\Delta} .
$$

A weighted harmonic mean interpolation is used to compute $\mu$ at cell face $(i+1 / 2, j)$ :

$$
\frac{1}{\mu_{i+1 / 2, j}}=\frac{1}{2}\left(\frac{1}{\mu_{i, j}}+\frac{1}{\mu_{i+1, j}}\right), \quad \frac{1}{\mu_{i, j}}=\frac{1-C_{i, j}}{\mu_{o}}+\frac{C_{i, j}}{\mu_{1}},
$$

where the discretized colour function $C_{i j}$ represents the volume fraction of the ferrofluid in cell $(i, j)$. Note that for large permeability ratios, the weighted harmonic mean is a better choice for the interpolation of the permeability for both cell and face values (Patankar 1980). Analogous relationships can be written for the other faces of a cell. A second-order discretization is used to discretize the Neumann condition for cells on the solid boundary. A multigrid solver is then used to obtain the solution of the resulting linear set of equations. 
The spatial discretization of the velocity field is based on the MAC grid in figure 22 . Therefore, the evaluation of the components of the magnetic stress tensor requires the evaluation of gradients at faces. In axisymmetric coordinates, the divergence of the magnetic stress tensor is discretized in the $\boldsymbol{e}_{r}$ - and $\boldsymbol{e}_{z}$-directions as

$$
\left.\begin{array}{l}
\frac{1}{r_{i+1 / 2, j}} \frac{r_{i+1, j}\left(\left(\boldsymbol{\tau}_{m}\right)_{r r}\right)_{i+1, j}-r_{i, j}\left(\left(\boldsymbol{\tau}_{m}\right)_{r r}\right)_{i, j}}{\Delta}+\frac{\left(\left(\boldsymbol{\tau}_{m}\right)_{r z}\right)_{i+1 / 2, j+1 / 2}-\left(\left(\boldsymbol{\tau}_{m}\right)_{r z}\right)_{i+1 / 2, j-1 / 2}}{\Delta}, \\
\frac{1}{r_{i, j+1 / 2}} \frac{r_{i+1 / 2, j+1 / 2}\left(\left(\boldsymbol{\tau}_{m}\right)_{r z}\right)_{i+1 / 2, j+1 / 2}-r_{i-1 / 2, j+1 / 2}\left(\left(\boldsymbol{\tau}_{m}\right)_{r z}\right)_{i-1 / 2, j+1 / 2}}{\Delta} \\
\quad+\frac{\left(\left(\boldsymbol{\tau}_{m}\right)_{z z}\right)_{i, j+1}-\left(\left(\boldsymbol{\tau}_{m}\right)_{z z}\right)_{i, j}}{\Delta},
\end{array}\right\}
$$

respectively, where, for example, components such as $\left(\left(\boldsymbol{\tau}_{m}\right)_{r r}\right)_{i, j}$ and $\left(\left(\boldsymbol{\tau}_{m}\right)_{r z}\right)_{i+1 / 2, j+1 / 2}$ are discretized as follows:

$$
\left.\begin{array}{rl}
\left(\left(\boldsymbol{\tau}_{m}\right)_{r r}\right)_{i, j}= & \mu_{i, j}\left[\left(\frac{\phi_{i+1, j}-\phi_{i-1, j}}{2 \Delta}\right)^{2}-\frac{1}{2}\left(\left(\frac{\phi_{i+1, j}-\phi_{i-1, j}}{2 \Delta}\right)^{2}\right.\right. \\
& \left.\left.+\left(\frac{\phi_{i, j+1}-\phi_{i, j-1}}{2 \Delta}\right)^{2}\right)\right], \\
\left(\left(\boldsymbol{\tau}_{m}\right)_{r z}\right)_{i+1 / 2, j+1 / 2}= & \mu_{i+1 / 2, j+1 / 2}\left(\frac{\phi_{i, j}-\phi_{i-1, j}+\phi_{i, j-1}-\phi_{i-1, j-1}}{2 \Delta}\right) \\
& \times\left(\frac{\phi_{i-1, j}-\phi_{i-1, j-1}+\phi_{i, j}-\phi_{i, j-1}}{2 \Delta}\right),
\end{array}\right\}
$$

respectively, where $\mu_{i+1 / 2, j+1 / 2}$ is computed using a simple averaging from cell centre values. Note that $\left(\boldsymbol{\tau}_{m}\right)_{r r}$ and $\left(\boldsymbol{\tau}_{m}\right)_{r z}$ at other grid locations are discretized similarly. Analogous relationships can be written for the other components of the magnetic stress tensor.

\section{REFERENCES}

Afkhami, S., Renardy, Y., Renardy, M., Riffle, J. S. \& St. Pierre, T. G. 2008a Field-induced motion of ferrofluid droplets through immiscible viscous media. J. Fluid Mech. 610, 363-380.

Afrhami, S., Renardy, Y., Renardy, M., Riffle, J. S. \& St. Pierre, T. G. $2008 b$ Numerical modeling of ferrofluid droplets in magnetic fields. In Proceedings of XVth International Congress on Rheology. American Institute of Physics.

Arkhipenko, V. I., Barkov, Y. D. \& Bashtovoi, V. G. 1978 Investigation of the form of a drop of a magnetizing liquid in a homogeneous magnetic field. Magnetohydrodynamics 14, 131-134.

BACRI, J. C. \& SALIN, D. 1982 Instability of ferrofluid magnetic drops under magnetic field. J. Phys. Lett. 43, 649-654.

Basaran, O. A. \& Wohlhuter, F. K. 1992 Effect of nonlinear polarization on shapes and stability of pendant and sessile drops in an electric (magnetic) field. J. Fluid Mech. 244, 1-16.

Blums, E., Cebers, A. \& Maiorov, M. M. 1997 Magnetic Fluids. De Gruyter.

Brackbill, J. U., Kothe, D. B. \& Zemach, C. 1992 A continuum method for modeling surface tension. J. Comput. Phys. 100, 335-354.

CEBers, A. 1982 Role of surface interactions in stratification of magnetic fluids. Magnetohydrodynamics 18, 345-350.

Cebers, A. 1985 Virial method of investigation of statics and dynamics of drops of magnetizable liquids. Magnetohydrodynamics 21, 19-26.

Cebers, A. 2002 The anisotropy of the surface tension at the magnetic-field-induced phase transformations. J. Magn. Magn. Mat. 252, 259-261. 
Dames, P., Gleich, B., Flemmer, A., Hajek, K., Seidl, N., Wiekhorst, F., Eberbeck, D., Bittmann, I., Bergemann, C., Weyh, T., Trahms, L., Rosenecker, J. \& Rudolph, C. 2007 Targeted delivery of magnetic aerosol droplets to the lung. Nature Nanotech. 2, 495-499.

Dodgson, N. \& Sozou, C. 1987 The deformation of a liquid drop by an electric field. J. Appl. Math. Phys. (ZAMP) 38, 424-432.

Drozdova, V. I., Skrobotova, T. V. \& Chekanov, V. V. 1979 Experimental study of the hydrostatics characterizing the interphase boundary in a ferrofluid. Magnetohydrodynamics 15, 12-14.

FenG, J. Q. \& ScOTT, T. C. 1996 A computational analysis of electrohydrodynamics of a leaky dielectric drop in an electric field. J. Fluid Mech. 311, 289-326.

Garton, C. G. \& Krasucki, Z. 1964 Bubbles in insulating liquids: stability in an electric field. Proc. R. Soc. Lond. A 280, 211-226.

Holligan, D. L., Gillies, B. T. \& Dailey, J. P. 2003 Magnetic guidance of ferrofluidic nanoparticles in an in vitro model of intraocular retinal repair. Nanotechnology 14 (6), 661-666.

Korlie, M. S., Mukherjee, A., Nita, B. G., Stevens, J. G., Trubatch, A. D. \& Yecko, P. 2008 Modeling bubbles and droplets in magnetic fluids. J. Phys.: Condens. Matter 20, 204143.

Lafaurie, B., Nardone, C., Scardovelli, R., Zaleski, S. \& Zanetti, G. 1994 Modelling merging and fragmentation in multiphase flows with SURFER. J. Comput. Phys. 113, 134-147.

Landau, L. D. \& Lifshitz, E. M. 1984 Electrodynamics of Continuous Media. Pergamon.

Lavrova, O., Matthies, G., Mitkova, T., Polevikov, V. \& Tobiska, L. 2006 Numerical treatment of free surface problems in ferrohydrodynamics. J. Phys.: Condens. Matter 18 (38), S2657-S2669.

Lavrova, O., Matthies, G., Polevikov, V. \& Tobiska, L. 2004 Numerical modeling of the equilibrium shapes of a ferrofluid drop in an external magnetic field. Proc. Appl. Math. Mech. 4, 704-705.

LI, J. \& RenARDY, Y. 1999 Direct simulation of unsteady axisymmetric core-annular flow with high viscosity ratio. J. Fluid Mech. 391, 123-149.

Li, J., Renardy, Y. \& Renardy, M. 2000 Numerical simulation of breakup of a viscous drop in simple shear flow through a volume-of-fluid method. Phys. Fluids 12, 269-282.

Liggieri, L., Sanfeld, A. \& Steinchen, A. 1994 Effects of magnetic and electric fields on surface tension of liquids. Physica A 206, 299-331.

Liu, X., Kaminski, M. D., Riffle, J. S., Chen, H., Torno, M., Finck, M. R., Taylor, L. \& Rosengart, A. J. 2007 Preparation and characterization of biodegradable magnetic carriers by single emulsion-solvent evaporation. J. Magn. Magn. Mat. 311, 84-87.

Markov, K. Z. 1999 Stability and instability in viscous fluids. In Heterogeneous Media: Modelling and Simulation (ed. K. Z. Markov \& L. Preziosi), chap. 1, pp. 1-162. Birkhäuser.

Martin, J. E., Venturini, E. L. \& Huber, D. L. 2008 Giant magnetic susceptibility enhancement in field-structured nanocomposites. J. Magn. Magn. Mat. 320, 2221-2227.

MefFord, O. T. 2007 Physical properties of macromolecule-metal oxide nanoparticle complexes: magnetophoretic mobility, size, and interparticle potentials. PhD thesis, Department of Chemistry, Virginia Tech.

Mefford, O. T., Carroll, M. R. J., Vadala, M. L., Goff, J. D., Mejia-Ariza, R., Saunders, M., Woodward, R. C., St. Pierre, T. G., Davis, R. M. \& Riffle, J. S. 2008 a Size analysis of PDMS-magnetite nanoparticle complexes: experiment and theory. Chem. Mater. 20 (6), 2184-2191.

Mefford, O. T., Vadala, M. L., Carroll, M. R. J., Mejia-Ariza, R., Caba, B. L., St. Pierre, T. G., Woodward, R. C., Davis, R. M. \& Riffle, J. S. $2008 b$ Stability of polydimethylsiloxanemagnetite nanoparticles against flocculation: interparticle interactions of polydisperse materials. Langmuir 24 (9), 5060-5069.

Mefford, O. T., Woodward, R. C., Goff, J. D., Vadala, T. P., St. Pierre, T. G., Dailey, J. P. \& Riffle, J. S. 2007 Field-induced motion of ferrofluids through immiscible viscous media: testbed for restorative treatment of retinal detachment. J. Magn. Magn. Mater. 311, 347-353.

Mendelev, V. S. \& Ivanov, A. O. 2004 Ferrofluid aggregation in chains under the influence of a magnetic field. Phys. Rev. E 70, 051502-1-051502-10.

Mikkelsen, C. I. 2005 Magnetic separation and hydrodynamic interactions in microfluidic systems. $\mathrm{PhD}$ thesis, Technical University of Denmark.

Miksis, M. J. 1981 Shape of a drop in an electric field. Phys. Fluids 24 (11), 1967-1972.

Patankar, S. V. 1980 Numerical Heat Transfer and Fluid Flow. McGraw-Hill. 
Rallison, J. M. 1980 Note on the time-dependent deformation of a viscous drop which is almost spherical. J. Fluid Mech. 98 (3), 625-633.

Rosensweig, R. E. 1985 Ferrohydrodynamics. Cambridge University Press.

SCARdovelli, R. \& Zaleski, S. 1999 Direct numerical simulation of free surface and interfacial flow. Annu. Rev. Fluid Mech. 31, 567-604.

ShahnaZian, H. \& Odenbach, S. 2007 Yield stress in ferrofluids? Intl J. Mod. Phys. B 21, 4806-4812.

SHERWOOD, J. D. 1991 The deformation of a fluid drop in an electric field: a slender-body analysis. J. Phys. A 24, 4047-4053.

Stone, H. A., Lister, J. R. \& Brenner, M. P. 1999 Drops with conical ends in electric and magnetic fields. Proc. R. Soc. Lond. A 455, 329-347.

TAYLOR, G. I. 1964 Disintegration of water drops in an electric field. Proc. R. Soc. Lond. A 280, 383-397.

Tyatyushin, A. N. \& Verlarde, M. G. 2001 On the interfacial deformation of a magnetic liquid drop under the simultaneous action of electric and magnetic fields. J. Colloid Intl Sci. 235, 46-58.

TYLER, A. J. 2008 Presentation: dynamic behaviour of magnetic fluid droplets in non-uniform magnetic fields.

Tyler, A. J., Woodward, R. C. \& St. Pierre, T. G. 2010 Variation in the surface energy of a ferrofluid droplet in an applied magnetic field (in preparation).

Voltairas, P. A., Fotiadis, D. I. \& Massalas, L. K. 2001 Elastic stability of silicone ferrofluid internal tamponade (SFIT) in retinal detachment surgery. J. Magn. Mag. Mat. 225, 248-255.

Voltairas, P. A., Fotiadis, D. I. \& Michalis, L. K. 2002 Hydrodynamics of magnetic drug targeting. J. Biomech. 35, 813-821.

Wohlhuter, F. K. \& Basaran, O. A. 1992 Shapes and stability of pendant and sessile dielectric drops in an electric field. J. Fluid Mech. 235, 481-510.

Woodward, R. C., Heeris, J., St. Pierre, T. G., Saunders, M., Gilbert, E. P., Rutnakornpituk, M., ZHANG, Q. \& RiffLe, J. S. 2007 A comparison of methods for the measurement of the particle-size distribution of magnetic nanoparticles. J. Appl. Cryst. 40, S495-S500. 